\title{
One-Dimensional String Theory with Vortices as Upside-Down Matrix Oscillator
}

\author{
Dmitri Boulatov \\ Institute of Theoretical and Experimental Physics \\ B.Cheremushkinskaya 25, 117259 Moscow, RUSSIA \\ and \\ Vladimir Kazakov \\ Laboratoire de Physique Théorique de l'Ecole Normale Supérieure, \\ 24, rue Lhomond, 75231 Paris Cedex 05, FRANCE
}

\begin{abstract}
We study matrix quantum mechanics at a finite temperature equivalent to one dimensional compactified string theory with vortex (winding) excitations. It is explicitly demonstrated that the states transforming under non-trivial $\mathrm{U}(\mathrm{N})$ representations describe various configurations vortices and anti-vortices. For example, for the adjoint representation the Feynman graphs (representing discretized world-sheets) contain two faces with the boundaries wrapping around the compactified target space which is equivalent to a vortex-anti-vortex pair. A technique is developed to calculate partition functions in a given representation for the standard matrix oscillator. It enables us to obtain the partition function in the presence of a vortex-anti-vortex pair in the double scaling limit using an analytical continuation to the upside-down oscillator. The Berezinski-Kosterlitz-Thouless phase transition occurs in a similar way and at the same temperature as in the flat $2 \mathrm{D}$ space. A possible generalization of our technique to any dimension of the embedding space is discussed.
\end{abstract}

LPTENS 91/24

KUNS 1094 HE(TH) 91/14

August 1991

‡ Unité de Recherche du Centre National de la Recherche Scientifique et de l'Ecole Normale Supérieure et à l'Université de Paris-Sud. 


\section{Introduction}

Matrix quantum mechanics represents an important analytical tool for the investigation of one dimensional bosonic string theory (see references in review [1]). Matrix formulation of this string theory is based on the correspondence between the feynman diagrammatic expansion of the matrix functional integral and the sum over all possible discretized (say, triangulated) curved two-dimensional manifolds embedded into one dimensional target space (time).

It was claimed in ref. [2] that the singlet sector of the quantum mechanical matrix model (trivial representation of $\mathrm{U}(\mathrm{N})$ ) compactified on a time circle of the length $\beta$ represents the vortex free sector of one dimensional bosonic string theory. This claim was supported by a remarkable self-duality with respect to the change $\beta \rightarrow \alpha \prime / \beta$ expected from the continuum string field theory.

Non-singlet states are of special interest in the $1 D$ matrix theory. It was conjectured in [2-3] that they correspond to the vortex-anti-vortex excitations (winding modes) of compactified $1 D$ bosonic string with the target space coordinate living on a circle of a finite length $\beta$. In the statistical mechanics language, they describe the classical $X Y$ model on a random (dynamical) 2D manifold.

In ref. [3] the simplest non-singlet case, (the adjoint representation for "angular" variables), was investigated. Using the results of the paper [4] where the planar $(N \rightarrow \infty)$ approximation for the adjoint hamiltonian was developed, the authors of ref. [3] have demonstrated that there exists a big gap separating ground states for the singlet and the adjoint representations:

$$
E_{\text {adj }}-E_{\text {sing }} \underset{\Delta \rightarrow 0}{\sim} \frac{\beta}{2 \pi}|\log \Delta|
$$

where $\Delta=\lambda_{c}-\lambda$ is the deviation of the cosmological constant from its critical value.

It was also argued in ref. [3] that this result can be qualitatively reproduced from the continuum Liouville theory assuming that one includes a vortex-anti-vortex pair into the system. So, the adjoint representation was conjectured to describe a vortex-anti-vortex pair in the presence of $2 \mathrm{D}$ quantum gravity. Higher representations were associated with bigger numbers of vortices. On the base of these conjectures the authors of ref. [3] gave an estimate of the position, $\beta_{c}$, of the Berezinski-Kostelitz-Thouless phase transition arguing that $\beta_{K T}$ is finite. The phase $\beta>\beta_{K T}$ (low vortex density) describes $c=1$ system and, for $\beta<\beta_{K T}, c=0$ (vortices destroy long order correlations). 
In this paper we shall use a different technique but the result (1.1) will be confirmed and even generalized. Namely, we shall calculate the double scaling limit of the partition function for the adjoint representation providing the partition function of vortexanti-vortex pair interacting with the $2 \mathrm{D}$ quantum gravity to all genera. We shall explicitly demonstrate by direct arguments that for the matrix quantum mechanics in the adjoint representation describes the "vortex-anti-vortex" sector of the $X Y$-model (sometimes called as the model of planar rotators) interacting with 2D quantum gravity. As in the $X Y$-model on a regular lattice, knowing the partition function in this sector one can determine $\beta_{K T}$ precisely. Our estimate $\beta_{K T}=4 \pi$ coincides with the one given in ref. [3].

We will propose here a mathematical setup, different from [3], for the analysis of nonsinglet sectors in the matrix quantum mechanics. Although we can solve a number of matrix models exactly we usually need from the solutions only a small piece of information about the continuum (thermodynamic) limit of lattice manifolds dominated by huge Feynman graphs. In this respect it seems to be very desirable to have in hands some formalism which has an advantage of the matrix approach to be manifestly invariant (i.e. not using the notion of a coordinate system and non-invariant objects like a metric) but avoiding the intermediate lattice stage usual for the matrix models and dealing directly with the continuum theory.

In the matrix language one usually diagonalizes the matrices and tries to formulate a model in terms of their eigenvalues integrating explicitly, if it appears to be possible, over the "angular" degrees of freedom belonging to a group $(\mathrm{U}(\mathrm{N})$ in the case of $N \times N$ Hermite-an matrices). For many interesting models of $2 \mathrm{~d}$ gravity with matter fields having the central charge $c \leq 1$, this integration can be performed exactly. In the resulting effective action for the eigenvalues, not the entire potential of the original matrix model is important for the continuum (planar or double scaling) limit but only the vicinity of a singularity of the effective action. In the most generic case, this is a quadratic top of the potential. The behavior of the eigenvalues near the top usually defines the essential, long distance phenomena and everything else in the shape of the potential is responsible only for microscopic details, i.e. cut-off, area definition etc. This brings to the mind the idea that the matrix models can be formulated in a simplified way where only the essential part of the matrix potential will be taken into account. One can hope that, in the continuum limit, the whole model might appear to be almost (i.e. up to a cut-off) Gaussian and, hence, exactly solvable even though the potential is unstable. 
One of the most transparent examples realizing this mechanism is the matrix quantum mechanics [5], which was shown to correspond to the bosonic string field theory in onedimensional target space [6] and was solved later in the double scaling limit [7-10]. This theory can be described by matrix quantum mechanics with an upside-down oscillatorial potential. The system can be stabilized by putting infinite walls on cut-off distances $\pm \Lambda$ from the top of the potential for every eigenvalue of the matrix field.

In this case it is natural to expect that interesting physical quantities can be obtained by an analytical continuation of corresponding quantities for the standard matrix oscillator with the stable quadratic potential, i.e. by the change $\omega \rightarrow i \omega$ (where $\omega$ is the frequency of the oscillator).

Life appears to be more complicated: the standard oscillator has more symmetry then the upside-down one and it does not know about any cut-off. Usually to establish a correspondence between them the direct analytical continuation is not possible and should be completed by a guess about the cut-off dependence. One of the recent results of this correspondence was given in ref. [11] for the singlet sector Green function where the corresponding planar result of ref. [12] was generalized to the double scaling limit.

In the present paper we give another example of such correspondence. Our goal will be to calculate partition functions for some non-singlet states in the $1 D$ model in the double scaling limit using partition functions for the ordinary matrix oscillator. We argue that this correspondence works at least for a sector of the model, given by the adjoint representation of $\mathrm{U}(N)$ and (as we show explicitly) describing single vortex-anti-vortex configuration. At the end we discuss a possible generalization of this approach to a $D+1$ dimensional matrix model which might give in the future new analytical tools into our hands.

The paper is organized as follows.

In section 2, we formulate the matrix model with the compactified time and discuss its correspondence with $1 D$ string theory and the $X Y$-model.

In section 3, we give a derivation of Hamiltonians in non-singlet sectors in terms of the eigenvalues of the matrix field. The double scaling is considered for which the problem can be reformulated as the upside-down matrix oscillator.

In section 4, a $U(N)$ twisted partition function is introduced which allows us to expand the oscillatorial partition function into a sum of partition functions of states in given representations of $U(N)$. In this section we also show that the adjoint sector of the 
$1 D$ matrix model corresponds to the insertion of one vortex-anti-vortex pair on the string world sheet.

In section 5, the analytical continuation of the adjoint partition function is suggested and, in section 6, physical consequences of the obtained results are considered.

In section 7, a possible generalization of our approach to the case of $D+1$ string theory is sketched out using the idea of correspondence between Gaussian matrix models with the stable and the upside-down quadratic potentials.

The section 8 is devoted to conclusion.

Three appendices contain technical details concerning applications of the group theory methods to our model.

\section{Matrix quantum mechanics in periodic time as 1D compactified string} theory

The matrix model under consideration can be defined by the following functional integral for the partition function:

$$
\mathcal{Z}_{N}(\beta, \lambda)=\int_{\varphi(0)=\varphi(\beta)} \mathcal{D}^{N^{2}} \varphi(t) \exp -N t r \int_{0}^{\beta} d t\left[\frac{1}{2} \dot{\varphi}^{2}+V(\varphi)\right]
$$

where $\varphi(t)$ is a hermitian-matrix-valued periodic $1 D$ field : $\varphi_{i j}(t)=\bar{\varphi}_{j i}(t) ; \varphi_{i j}(0)=\varphi_{i j}(\beta)$ $i, j=1, \ldots, N$. The potential can be chosen, for example, in the form

$$
V(\varphi)=\frac{1}{2} \varphi^{2}-\frac{\lambda}{3} \varphi^{3}
$$

which corresponds to triangulations of the string world sheet.

The standard arguments leading to the string picture interpretation of eq. (2.1) are the following [3]. Let us expand $\log \mathcal{Z}_{N}(\beta, \lambda)$ in the coupling $\lambda$ using diagrammatic Feynman rules. The corresponding free energy $f_{N}(\beta, \lambda)=\frac{1}{N^{2}} \log \mathcal{Z}_{N}(\beta, \lambda)$ has the form:

$$
\begin{aligned}
f_{N}(\beta, \lambda)= & \sum_{g=0}^{\infty} N^{-2 g} \sum_{V=0}^{\infty} \lambda^{V} \sum_{G_{g}^{(V)}} \int_{0}^{\beta} \cdots \int_{0}^{\beta} d t_{1} \cdots d t_{V} \times \\
& \prod_{<i j>m_{i j}=-\infty} \sum^{\infty} e^{-\left|t_{i}-t_{j}+\beta m_{i j}\right|}
\end{aligned}
$$


where $g$ is the genus of a $\varphi^{3}$-Feynman graph $G_{g}^{(V)} ; V$ is the number of vertices, $\prod_{<i j>}$ is the product over all links of the graph, $<i j>\in G_{g}^{(n)}$, and the periodic propagator in our case is

$$
D\left(t_{i}-t_{j}\right)=\sum_{m=-\infty}^{\infty} e^{-\left|t_{i}-t_{j}+m \beta\right|}
$$

where $t_{i}$ 's are attached to vertices of a graph. The constant $\lambda$ plays the role of a bare cosmological constant and $1 / N$ is the (topological) string coupling constant. For sufficiently large $\beta$ we expect that only the term with $m=0$ in eq. (2.4) will survive and we will come to the well investigated case of the $1 D$ matrix model on the infinite time interval [1]. In this case time coordinates $t_{i}, i=1,2 \ldots V$, will correspond to the target space coordinates of the string world sheet represented by a $\varphi^{3}$-graph $G_{g}^{(V)}$. The corresponding action is of the form

$$
S=\sum_{<i j>\in G_{g}^{(V)}}\left|t_{i}-t_{j}\right|
$$

which differs from the discretized Polyakov string action :

$$
S_{p}=\sum_{<i j>\in G_{g}^{(V)}}\left(t_{i}-t_{j}\right)^{2}
$$

but is proven to be in the same universality class due to the superconvergency of underlying Feynman diagrams.

Eq. (2.3) describes the $X Y$-model coupled to $2 d$ gravity and we expect the appearance of all phenomena related to the vortex configurations of the $t$-field. The analysis of the vortex kinematics in the model was done in ref. [2]. Let us briefly mention it here for completeness. Following the classical papers [13],[14], [15] we can view $m_{i j}$ in eq.(2.3) as an abelian gauge field defined on links of a $\varphi^{3}$-graph. The sum along a non-self-intersecting loop $L$ on the graph

$$
M_{L}=\sum_{<i j>\in L} m_{i j}
$$

gives the integral charge of the vortices enveloped by the contour $L$. In the case when $L$ is just the boundary of a face of the graph, $M_{L}$ can be considered as the elementary field strength or the vortex number in this face. The duality transformation corresponding to the Fourier transform of the original propagators

$$
\sum_{m=-\infty}^{\infty} e^{-\left|t_{i}-t_{j}+\beta m\right|}=\frac{2}{\beta} \sum_{k_{i j}=-\infty}^{\infty} e^{i \frac{2 \pi}{\beta} k_{i j}\left(t_{i}-t_{j}\right)} \frac{1}{1+\left(\frac{2 \pi}{\beta} k_{i j}\right)^{2}}
$$


brings eq. (2.3) to the form where $M_{L}$ are new dynamical variables. If we insert eq. (2.8) into eq. (2.3) and integrate over $t_{i}$ 's, we obtain the constraint at every vertex $i$, which is just the ordinary condition of the momentum conservation,

$$
k_{i j_{1}}+k_{i j_{2}}+k_{i j_{3}}=0
$$

It means that (excluding the zero modes) we have got $E-V+1=F-1+2 g$ variables instead of $V-1$ (in virtue of the Euler theorem: $F-E+V=2-2 g$, where $F, E$ and $V$ are the numbers of faces, edges and vertices of a graph). The usual choice of these variables is to attach a momentum $p_{I}$ to each face and to define remaining $2 g$ variables as momenta $l_{a}$ running along $2 g$ independent non-contractable loops on a graph: $L_{a}, a=1, \ldots, 2 g$.

The resulting dual representation for the partition function (2.3) takes the form

$$
\begin{aligned}
f_{N}(\beta, \lambda)= & \left(\frac{\beta}{\lambda}\right)^{2} \sum_{g=0}^{\infty}\left(\frac{N \beta}{\lambda}\right)^{-2 g} \sum_{F}\left(\frac{\lambda^{\frac{1}{2}}}{\beta}\right)^{F} \sum_{\tilde{G}_{g}^{(F)}} \sum_{p_{1}=-\infty}^{+\infty} \ldots \sum_{p_{F}=-\infty}^{+\infty} \\
& \sum_{l_{1}=-\infty}^{+\infty} \ldots \sum_{l_{2 g}=-\infty}^{+\infty} \prod_{<I J>} \frac{2}{1+\left(\frac{2 \pi}{\beta}\right)^{2}\left(p_{I}-p_{J}+\sum_{a=1}^{2 g} l_{a} \epsilon_{I J}^{a}\right)^{2}}
\end{aligned}
$$

where $\sum_{\tilde{G}_{g}^{(F)}}$ is the sum over all dual graphs (triangulations) having $F$ dual vertices and a genus $g ; \prod_{<I J>}$ is the product over all dual links $\left\langle I J>\in \tilde{G}_{g}^{(F)} ; \epsilon_{I J}^{a}= \pm 1\right.$ when a dual edge $\langle I J\rangle$ crosses an edge belonging to a chosen in advance non-contractable cycle $L_{a}$, and is zero otherwise. The sign has to be chosen with respect to the mutual orientation of the link $\left\langle I J>\right.$ and the loop $L_{a}$.

It can be argued that one can discard the vortices in eq.(2.3) by imposing the "pure gauge" conditions on the field $m_{i j}$ (up to a contribution of non-contractable loops)

$$
m_{i j}=\sum_{a=1}^{2 g} \tilde{\epsilon}_{i j}^{a} \tilde{l}_{a}+m_{i}-m_{j}
$$

where integers $\tilde{l}_{a}$ are defined on non-contractable dual loops $\tilde{L}_{a} ; \tilde{\epsilon}_{i j}^{a}$ is the object dual to $\epsilon_{I J}^{a}$. Then, eq. (2.10) will look as

$$
\begin{aligned}
& f_{N}(\beta, \lambda)=\left(\frac{\beta}{\lambda}\right)^{2} \sum_{g=0}^{\infty}\left(\frac{N \beta}{\lambda}\right)^{-2 g} \sum_{F}\left(\frac{\lambda^{\frac{1}{2}}}{\beta}\right)^{F} \sum_{\tilde{G}_{g}^{(F)}} \\
& \int_{-\infty}^{\infty} \prod_{I=1}^{F} d p_{I} \sum_{l_{1}=-\infty}^{\infty} \ldots \sum_{l_{2 g}=-\infty}^{+\infty} \prod_{<I J>} \frac{2}{1+\left(\frac{2 \pi}{\beta}\right)^{2}\left(p_{I}-p_{J}+\sum_{a=1}^{2 g} l_{a} \epsilon_{I J}^{a}\right)^{2}}
\end{aligned}
$$


The sums over $p_{I}$ in eq. (2.10) are substituted by the integrals, since, in the original expression (2.3) with the choice (2.11), we can instead of summing over $m_{i}$ 's spread the integrations over $t_{i}$ 's to the infinite interval. Then the corresponding Fourier transforms of propagators will be integrals rather than sums.

Now the original representation of $f_{N}(\beta, \lambda)$, eq. (2.3), with the condition (2.11) looks very similar to its dual transform (2.12). The arguments of the propagators and the string coupling constant can be matched by the simple duality transformation

$$
\begin{aligned}
& \frac{\beta}{2 \pi} \rightarrow \frac{2 \pi}{\beta} \\
& \frac{1}{N} \rightarrow \frac{2 \pi}{\beta N}
\end{aligned}
$$

Two main differences are : the original $\varphi^{3}$-graphs are substituted by dual ones, and the propagators are different. If we believe in universality in the continuum limit, these two differences should be insignificant on the macroscopic scale. More important is the

equivalence of the sets of variables $\left(t_{i}, \tilde{l}_{a}\right)$ and $\left(p_{I}, l_{a}\right)$ in eqs. (2.3) and (2.12). So, one can hope that, in the double scaling limit, all answers will be invariant under the duality transformation (2.13). It was claimed in ref. [2] that this is really true for the singlet sector of the model, which was identified therefore with the vortex-free partition function (2.12). We will show in this paper that the inclusion of the non-singlet states destroys the self-duality, which is natural to expect in the presence of vortices.

\section{Effective action for eigenvalues}

The first standard step to calculate the partition function (2.1) is the diagonalization of the matrix field:

$$
\varphi_{i j}(t)=\sum_{k=1}^{N} \Omega_{i k}^{+}(t) z_{k}(t) \Omega_{k j}(t)
$$

where $\Omega(t) \in \mathrm{U}(\mathrm{N})$.

In terms of new variables we have

$$
\operatorname{tr} \dot{\varphi}^{2}=\sum_{i=1}^{N} \dot{z}_{i}^{2}+\sum_{i \neq j}\left(z_{i}-z_{j}\right)^{2}\left|A_{i j}\right|^{2}
$$

where we introduced "the connection"

$$
A_{i j}(t)=\left(\Omega^{+} \dot{\Omega}\right)_{i j}
$$


The periodicity condition $\varphi_{i j}(t)=\varphi_{i j}(t+\beta)$ implies that eigenvalues, $z_{k}$, have to be periodic in $t$ only up to an arbitrary substitution $\mathcal{P}$ :

$$
\begin{array}{r}
z_{k}(t+\beta)=\sum_{j=1}^{N} \mathcal{P}_{k j} z_{j}(t) \mathcal{P}_{j k}^{-1} \\
\Omega(t+\beta)=\mathcal{P} \Omega(t)
\end{array}
$$

which shows that the variables $A_{i j}(t)$ are not independent, contrary to the free boundary conditions case, and that they obey the constraint:

$$
\widehat{T} \exp i \int_{0}^{\beta} d t A(t)=\mathcal{P}^{-1}
$$

This makes the integral over $A(t)$ rather complicated unlike the most of solvable models of $2 d$ gravity with $c<1$. Usually, attempts to solve some matrix models describing gravity with matter having the central charge $c>1$ are blocked by the difficulties to compute the emerging integrals over the "angular" variables. We hope that the investigation of the model containing at least one nontrivial integration might be useful for the understanding of the role of "angular" variables for $c>1$.

The Dyson measure for the new variables $z$ and $\Omega$ looks as

$$
\begin{aligned}
\mathcal{D} \varphi(t) & =\prod_{t \in[0, \beta]} \Delta^{2}(z(t)) \prod_{k=1}^{N} d z_{k}(t) d^{N^{2}} A(t) \times \\
& \times \delta_{U(N)}\left(\widehat{T} \exp \int_{0}^{\beta} A d t, \mathcal{P}^{-1}\right)
\end{aligned}
$$

where $\Delta(z)=\prod_{k>m}\left(z_{k}-z_{m}\right)$ is the Vandermonde determinant; the invariant $\delta$-function can be represented as sum over irreducible representations

$$
\delta_{U(N)}\left(\Omega, \mathcal{P}^{-1}\right)=\sum_{R} d_{R} \chi_{R}(\mathcal{P} \Omega)
$$

where $d_{R}$ is the dimension of the $\mathrm{R}$ 'th representation and $\chi_{R}(\Omega)$ is the character

$$
\chi_{R}\left(\widehat{T} \exp i \int_{0}^{\beta} A d t\right)=\operatorname{Tr}_{R} \widehat{T} \exp \left(i \int_{0}^{\beta} d t \sum_{i, j} A_{i j} \widehat{\tau}_{i j}^{R}\right),
$$

where $\hat{\tau}_{i j}^{R}$ is a generator of $U(N)$ in the R'th representation.

It is well known [1] that in the case of free boundary conditions (i.e. $\varphi(0)$ independent of $\varphi(\beta))$ the singlet partition function describes non-interacting fermions. Indeed after 
integration over all angular variables only two Vandermonde determinants at the ends of the interval remain: $\Delta(z(0)) \Delta(z(\beta))$ and assure the antisymmetry of wave functions. In the case of the periodic boundary conditions, one should be more careful and use $\delta$-function to match the eigenvalues:

$$
f(z(\beta))=\int \prod_{k} d z_{k}(0) \Delta^{2}(z(0)) f(z(0)) \delta(z(0)-z(\beta))
$$

Substituting eqs. (3.1), (3.6) and (3.8) in eq.(2.1) we obtain after integration over $A(t)$ a functional integral over eigenvalues

$$
\begin{aligned}
& \mathcal{Z}_{N}(\beta, \lambda)=\frac{1}{N !} \sum_{\{\mathcal{P}\}}(-1)^{\mathcal{P}} \int \prod_{k=1}^{N} \mathcal{D} z_{k}(t) e^{-S_{0}\left(z_{k}\right)} \\
& \sum_{R} d_{R} \operatorname{tr}_{R}\left\{\widehat{T} \exp \left(\frac{1}{4 N} \int_{0}^{\beta} d t \sum_{i<j} \frac{\left(\hat{\tau}_{i j}^{R} \hat{\tau}_{j i}^{R}\right)}{\left(z_{i}-z_{j}\right)^{2}}\right) \mathcal{P}\right\}
\end{aligned}
$$

where $\frac{1}{N !} \sum_{\{\mathcal{P}\}}(-1)^{\mathcal{P}}$ is a standard anti-symmetrizator, which appeared because of the skew-symmetry of the Vandermonde determinant:

$$
\Delta\left(\mathcal{P} z(0) \mathcal{P}^{-1}\right)=(-1)^{\mathcal{P}} \Delta(z(0))
$$

and $S_{0}$ is the representation independent part of the action:

$$
S_{0}(z)=N \int_{0}^{\beta} d t\left[\frac{\dot{z}^{2}}{2}+V(z)\right]
$$

The $\widehat{T}$-ordering acts in the space of a representation $\mathrm{R}\left(\tau_{i j}^{R} \tau_{j i}^{R}\right.$ is the matrix product in this space). As it follows from eq. (3.2), the action does not contain a quadratic part for the diagonal elements of the field $A_{i j}$. The integration over them gives rise to a selection rule for representations. It is shown in the Appendix A that it selects only such $R$ for which the sum of all components of the highest weight,

$$
\begin{array}{r}
R=\left[m_{1}, m_{2} \cdots m_{N}\right] \\
m_{1} \geq m_{2} \cdots \geq m_{N},
\end{array}
$$

equals to zero $\dagger$ :

$$
\sum_{k=1}^{N} m_{k}=0
$$

$\dagger$ Let us note here that this class of allowed representations is much wider than the class of the self-conjugate ones (i.e. such that $m_{N-n}=N-m_{n}$ ) which was considered in the paper [3]. 
We conclude from eq. (3.10) that $\mathcal{Z}_{N}(\beta, \lambda)$ can be represented in the form of Gibbs partition function:

$$
\mathcal{Z}_{N}(\beta, \lambda)=\sum_{R} d_{R} \operatorname{Tr}_{R} e^{-\beta \widehat{H}_{R}}
$$

where the Hamiltonians $\widehat{H}_{R}$ are given by

$$
H_{R}=P_{R} \sum_{k=1}^{N}\left[-\frac{1}{2 N} \frac{\partial^{2}}{\partial z_{k}^{2}}+N V\left(z_{k}\right)\right]+\frac{1}{4 N} \sum_{i<j} \frac{\widehat{\tau}_{i j}^{R} \widehat{\tau}_{i j}^{R}}{\left(z_{i}-z_{j}\right)^{2}}
$$

$P_{R}$ defined by eq. (A5) is a projector onto the subspace of all zero weight vectors in the space of representation $\mathrm{R}$ (i.e. the kernel space of the generators of the Cartan subalgebra). The sum in eq. (3.15) runs over all irreducible representations obeying the selection rule (3.14). Only for such representations this subspace is not empty.

We see that unlike the simplest case of the trivial representation the eigenvalues cannot be considered here as non-interacting fermions (see Appendix A where transformational properties of $\Psi$-functions under permutations of eigenvalues are described).

A natural question arises whether, for higher representations, the same double scaling limit is applicable as for the trivial one, since the interaction among eigenvalues could completely change the critical properties of the system. However, we hope that, if the higher representations really correspond to the implementation of vortices, the critical properties of the world sheet of string are still the same as without vortices, at least far away from a vortex position. So, it is conceivable to adopt the same double scaling procedure as in [1]. It is based on the fact that the potential $\mathrm{V}(\mathrm{z})$ has a shape as shown in fig.1. It has a well filled by eigenvalues, which are kept there since the tunneling through the barrier is exponentially small with respect to its width. The decay of this system is not essential at least in every order of the $\frac{1}{N}$ (topological) expansion. The instability corresponding to the dominance of large graphs (thermodynamical limit) emerges when the eigenvalues near the Fermi level begin to spill over the top of the barrier, which can be achieved by tuning either $\lambda$ or $\mathrm{N}$. 


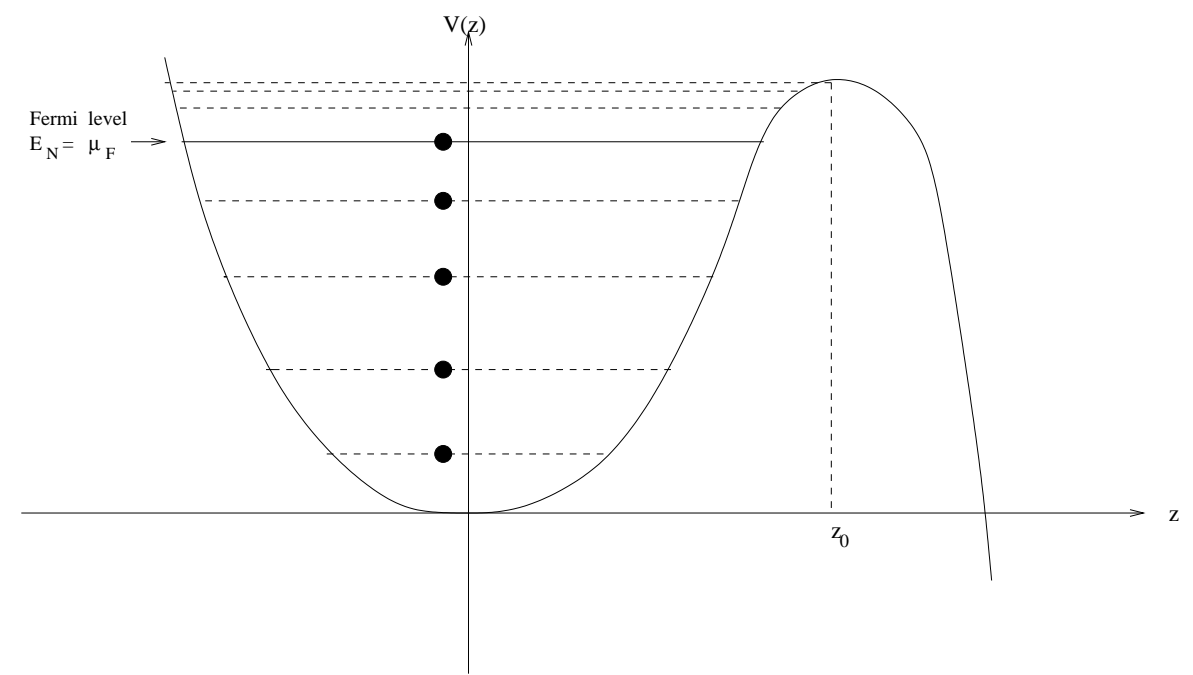

Fig. 1: A typical potential $V(z)$ for an eigenvalue $z$ in eq.(3.12). The ground state is fermionic, i.e. first $N$ quasi-stable levels in this unstable potential are occupied. The criticality appears when the Fermi level $\mu_{F}$ touches the local maximum at $z=z_{0}$.

This shows that one has to concentrate oneself on the vicinity of the top which defines all the interesting physics. Let us choose new coordinates

$$
x_{i}=\sqrt{N}\left(z_{i}-z_{0}\right)
$$

where $z_{0}$ is the position of the top $\left(z_{0}=\frac{1}{\lambda}\right.$ for the cubic potential (2.2)). Then

$$
V(z(x))=-\frac{1}{2 N} x^{2}+\sum_{k \geq 3} \frac{C_{k}}{N^{k / 2}} x^{k}
$$

all the powers of $X^{k}, k \geq 3$, are suppressed by the negative powers of $\mathrm{N}$ and can be omitted in the double scaling limit. Therefore, the essential part of the Hamiltonian (3.16) is

$$
H_{R}=P_{R} \sum_{k=1}^{N}\left[-\frac{1}{2} \frac{\partial^{2}}{\partial x_{k}^{2}}-\frac{1}{2} x_{k}^{2}\right]+\frac{1}{2} \sum_{i \neq j} \frac{\widehat{\tau}_{i j}^{R} \widehat{\tau}_{i j}^{R}}{\left(x_{i}-x_{j}\right)^{2}}
$$

Of course, this Hamiltonian is ill defined because of the unstable quadratic potential and at some moment we have to remember about higher powers of $x$ in eq. (3.18). The cubic term in eq. (3.18) provides an infinite wall at the distance

$$
\Lambda \sim \sqrt{N}
$$

from the top of the potential. 
As was noticed in ref. [1], one can define a completely stable system having the same $1 / \mathrm{N}$-expansion by putting two symmetric walls at distances $\pm \Lambda$ from the top, i.e. our system can be stabilized by putting it into the $\mathrm{N}$-dimensional hyper-cubic box $\{-\Lambda<$ $\left.x_{i}<\Lambda, i=1, \cdots N\right\}$. The convenience of the eigenvalue representation (3.19) is based on the fact that every eigenvalue has independent boundary condition. However, unlike the singlet representation, the eigenvalues are now interacting.

On the other hand, it is obvious that eq. (3.19) can be viewed as the eigenvalue representation of the matrix upside-down oscillator with the Hamiltonian:

$$
\widehat{H}(\varphi)=-\frac{1}{2} \operatorname{tr}\left(\frac{\partial^{2}}{\partial \varphi^{2}}+\omega^{2} \varphi^{2}\right)
$$

where we have introduced the frequency $\omega$ by simple rescaling of the time. On the first glance, eq. (3.21) represents the system of $N^{2}$ non-interacting oscillators

$$
H(\varphi)=-\frac{1}{2} \sum_{i>j}\left(\frac{\partial^{2}}{\partial \varphi_{i j} \partial \varphi_{i j}^{*}}\right)-\frac{1}{2} \sum_{k=1}^{N}\left(\frac{\partial^{2}}{\partial \varphi_{k k}^{2}}\right)
$$

But the boundary conditions, which are determined now by higher terms of the type

$$
\operatorname{tr} \varphi^{3}=\sum_{i, j, k} \varphi_{i j} \varphi_{j k} \varphi_{k j}
$$

mix all matrix elements and are not so simple as those for eigenvalues.

\section{Oscillatorial partition functions in different representations.}

The results of the previous section show that we can view one dimensional bosonic string theory as a theory of the upside down matrix oscillator, eq. (3.21), with U(N)symmetric stabilizing walls (cut-off) at a large distance $(\sim \Lambda)$ from the top of the potential.

Formally, if we forget for a while about the cut-off, this system is related to the standard oscillator with the stable matrix potential by simple analytical continuation to the imaginary frequency:

$$
\omega \rightarrow i \omega
$$

We might hope that physical quantities for the standard oscillator such as the partition function, Green's functions etc. already contain some information about the upside down oscillator. 
But if we take, for example, the partition function for the standard oscillator, which is equal to the product of partition functions of all matrix elements:

$$
Z^{(N)}(q)=\left(\frac{q^{1 / 2}}{1-q}\right)^{N^{2}}
$$

where

$$
q=e^{-\beta \omega}
$$

we see that it is too simple to be able to describe all the complexity of physics of 1dimensional strings even after an analytical continuation. Apparently, the information about the cut-off is lost in (4.2), and we have to try more suitable quantities in order to get some information about the upside-down oscillator.

A natural idea arises to classify all the states of the oscillator with respect to the irreducible representations of the $\mathrm{U}(\mathrm{N})$ rotations of the matrix coordinate:

$$
\varphi \rightarrow \Omega^{+} \varphi \Omega
$$

The corresponding wave functions $\Psi_{R}(\varphi)$ of these states should transform as matrix elements in a given representation R. Being formulated as a function of eigenvalues of $\varphi$ this wave function obeys the Schrödinger equation:

$$
\widehat{H}_{R} \Psi_{R}(z)=E \Psi_{R}(z)
$$

with $\widehat{H}_{R}$ given by eq.(3.19).

The partition function in the $\mathrm{R}$ 'th representation will be given by

$$
Z_{R}^{(N)}=\operatorname{Tr} e^{-\beta \widehat{H}_{R}}
$$

As we shall see in the next section, this quantity contains much more information about the continuation to the upside-down oscillator. The success of this approach is based on the fact that the cut-off wall for the upside-down oscillator is defined by higher $\mathrm{U}(\mathrm{N})$ invariant terms in the matrix potential (such as $\lambda_{1} \operatorname{tr} \varphi^{3}+\lambda_{2} \operatorname{tr} \varphi^{4}+\cdots$ ), and therefore it does not destroy the classification of states in representations: every wave in a given representation $R$ being sent to the wall will be reflected with the same properties under $\mathrm{U}(\mathrm{N})$ transformations. Hence, we think that the analytical continuation procedure should be separate for every representation.

Let us now work out the framework of finding $Z_{R}^{(N)}$ for the standard oscillator. 
The most elegant way to do it is to define the so called twisted partition function:

$$
Z^{(N)}(\Omega)=\operatorname{Tr}\left(e^{-\beta \widehat{H}} \widehat{\Theta}(\Omega)\right)=\int d e \rho_{\Omega}(E) e^{-\beta E}
$$

where $\widehat{\Theta}(\Omega)$ is a twist operator which "rotates" the final states with respect to the initial ones by the $\mathrm{U}(\mathrm{N})$ matrix $\Omega$ and

$$
\rho_{\Omega}(E)=\frac{1}{\pi} \operatorname{Im} \sum_{E^{\prime}} \int d^{N^{2}} \varphi \frac{\Psi_{E^{\prime}}^{*}(\varphi) \Psi_{E^{\prime}}\left(\Omega^{+} \varphi \Omega\right)}{E-E^{\prime}+i \varepsilon}
$$

can be called the "twisted density of states" though it does not have even normal properties of a density (say, it is not positively defined).

Then we can represent the eq. (4.7) as

$$
Z^{(N)}(\Omega)=\sum_{R} d_{R} Z_{R}^{(N)} \chi_{R}(\Omega)
$$

and for the twisted density of states:

$$
\rho_{\Omega}(E)=\sum_{R} d_{R} \rho_{R}(E) \chi_{R}(\Omega)
$$

where the characters $\chi_{R}(\Omega)$ obey the orthogonality relation:

$$
\int(d \Omega) \chi_{R_{1}}\left(\Omega^{+}\right) \chi_{R_{2}}(\Omega \cdot \omega)=\delta_{R_{1} R_{2}} \chi_{R_{1}}(\omega)
$$

and hence

$$
Z_{R}^{(N)}=\int(d \Omega) \chi_{R}(\Omega) Z^{(N)}(\Omega)
$$

and

$$
\rho_{R}^{(N)}(E)=\int(d \Omega) \chi_{R}(\Omega) \rho_{\Omega}(E)
$$

The simplest way to make these formulae more explicit is to use the Green function defined by the equation:

$$
\left[\frac{\partial}{\partial \beta}-\operatorname{tr}\left(\frac{1}{2} \frac{\partial^{2}}{\partial \varphi^{2}}-\frac{\omega^{2}}{2} \varphi^{2}\right)\right] G\left(\beta, \varphi, \varphi^{\prime}\right)=0
$$

with the initial condition:

$$
G\left(0, \varphi, \varphi^{\prime}\right)=\delta^{\left(N^{2}\right)}\left(\varphi-\varphi^{\prime}\right)
$$


The solution is well known to be

$$
G\left(\beta, \varphi, \varphi^{\prime}\right)=\left(\frac{\omega}{2 \pi \operatorname{sh} \omega \beta}\right)^{N^{2} / 2} \exp \left\{-\frac{\omega}{2} \operatorname{cth} \omega \beta \operatorname{tr}\left(\varphi^{2}+\varphi^{\prime 2}\right)+\frac{\omega}{\operatorname{sh} \omega \beta} \operatorname{tr}\left(\varphi \varphi^{\prime}\right)\right\}
$$

After the simultaneous change

$$
\begin{aligned}
& \omega \rightarrow i \omega \\
& \beta \rightarrow i \beta
\end{aligned}
$$

eq.(4.16) can be viewed as the Green's function for the upside-down matrix oscillator.

Eq.(4.16) appears to be a very useful starting point to many problems related to the one-dimensional strings. Let us, for example, demonstrate that the singlet sector of this theory, i.e. the sector bound to operators of the type

$$
O_{\alpha}(t)=\operatorname{tr} e^{\alpha \varphi(t)}=\sum_{k=1}^{N} e^{\alpha z_{k}(t)}
$$

which are invariant functions of $\varphi$ depending only on eigenvalues, can be described in terms of non-interacting fermions.

Indeed, if we calculate the two-point function, $D_{\alpha_{1} \alpha_{2}}(\beta)$, we obtain:

$$
\begin{aligned}
& D_{\alpha_{1} \alpha_{2}}(\beta)=\ll \operatorname{tr} e^{\alpha_{1} \varphi(0)} \operatorname{tre}^{\alpha_{2} \varphi(\beta)} \gg= \\
& \int d^{N^{2}} \varphi \int d^{N^{2}} \varphi^{\prime} \operatorname{tr} e^{\alpha_{1} \varphi} \operatorname{tre}^{\alpha_{2} \varphi^{\prime}} G\left(\beta, \varphi, \varphi^{\prime}\right)= \\
& \left(\frac{\omega}{2 \pi s h \omega \beta}\right)^{\frac{N^{2}}{2}} \sum_{k, m} \int \prod_{i=1}^{N} d z_{i} d z_{i}^{\prime} \exp \left\{-\frac{\omega}{2} \operatorname{cth} \omega \beta\left(z_{i}^{2}+z_{i}^{\prime 2}\right)\right\} \\
& \times e^{\alpha_{1} z_{k}+\alpha_{2} z_{m}^{\prime}} \times \Delta^{2}(z) \Delta^{2}\left(z^{\prime}\right) \int(d \Omega) \exp \left\{\frac{\omega}{\operatorname{sh} \omega \beta} \sum_{\alpha, b} \Omega_{a b}^{+} z_{b}^{\prime} \Omega_{b a} z_{a}\right\} \\
& =\left(\frac{\omega}{2 \pi s h \omega \beta}\right)^{\frac{N^{2}}{2}} \sum_{k, m} \int \prod_{i=1}^{N} d z_{i} d z_{i}^{\prime} \exp \left\{-\frac{\omega}{2} \operatorname{cth} \omega \beta\left(z_{i}^{2}+z_{i}^{\prime 2}\right)+\frac{\omega}{\operatorname{sh} \omega \beta} z_{i} z_{i}^{\prime}\right\} \\
& \times \Delta(z) \Delta\left(z^{\prime}\right) e^{\alpha_{1} z_{k}+\alpha_{2} z_{m}^{\prime}}
\end{aligned}
$$

where we used the "angular" decomposition of the matrices $\varphi=\omega^{+} z \omega$ and $\varphi^{\prime}=(\Omega \omega)^{+} z^{\prime}(\Omega \omega)$ and the well-known formula [16] :

$$
\int(d \Omega) \exp a \operatorname{tr}\left(\Omega^{+} z^{\prime} \Omega z\right)=\left(\prod_{k=1}^{N-1} k !\right) a^{-\frac{N(N-1)}{2}} \frac{\operatorname{det}_{k m}\left(e^{a z_{k}^{\prime} z_{m}}\right)}{\Delta\left(z^{\prime}\right) \Delta(z)}
$$


We see from eq. (4.20) that the initial and final states in terms of eigenvalues appear to be completely anti-symmetric due to the Vandermonde determinants $\Delta\left(z^{\prime}\right)$ and $\Delta(z)$. Hence, they can be represented as Slater determinants of the eigenfunctions of $\mathrm{N}$ oscillators (or upside-down oscillators):

$$
\Psi_{n_{1} n_{2} \cdots n_{N}}\left(z_{1}, \cdots z_{N}\right)=\frac{1}{\sqrt{N !}} \underset{(i, j)}{\operatorname{det}} \Psi_{n_{i}}\left(z_{j}\right)
$$

So, we deal with free fermions. In principle, starting from here we can obtain all the results of the paper [11] for the two-point function in the double scaling regime.

On the other hand, if we take the two point function of another type

$$
\begin{aligned}
K_{\alpha_{1} \alpha_{2}}^{(\beta)} & =<\frac{t r}{N}\left[O_{\alpha_{1}}(0) O_{\alpha_{2}}(\beta)\right]> \\
& =\int d^{N^{2}} \varphi d^{N^{2}} \varphi^{\prime} \frac{\operatorname{tr}}{N}\left[e^{\alpha_{1} \varphi} e^{\alpha_{2} \varphi^{\prime}}\right] G\left(\beta, \varphi, \varphi^{\prime}\right)
\end{aligned}
$$

we have a more complicated integral over $\mathrm{U}(\mathrm{N})$ group of the type

$$
\int(d \Omega) \Omega_{i j}^{+} \Omega_{j i} \exp \left\{a \operatorname{tr}\left(\Omega^{+} z^{\prime} \Omega z\right)\right\}
$$

Since the matrix element of the adjoint representation $D_{i j}^{k \ell}$ looks as

$$
D_{k \ell}^{i j}=\left(\Omega^{+}\right)_{j i} \Omega_{k \ell}-\frac{1}{N} \delta_{k}^{i} \delta_{\ell}^{j}
$$

we expect that the Green's function (4.23) describes the propagation of states transforming under the adjoint representation of $\mathrm{U}(\mathrm{N})$. Indeed, one can show (see Appendix B) that

$$
K_{\alpha_{1} \alpha_{2}}(\beta)=\sum_{m, n}\left\langle 0\left|\left(e^{\alpha_{1} z_{m}}\right)\left(e^{-\beta \widehat{H}_{a d j}}\right)_{m n}^{m n}\left(e^{\alpha_{2} z_{n}}\right)\right| 0\right\rangle
$$

where

$$
\begin{aligned}
& \left(\widehat{H}_{a d j}\right)_{i j}^{m n}=\delta^{m n} \delta_{i j} \sum_{k}\left(-\frac{1}{2} \frac{\partial^{2}}{\partial z_{k}^{2}}-\frac{\omega^{2}}{2} z_{k}^{2}\right)+ \\
& +\frac{1}{2} \delta^{m n} \delta_{i j}\left(\sum_{k \neq i} \frac{1}{\left(z_{i}-z_{k}\right)^{2}}+\sum_{k \neq n} \frac{1}{\left(z_{k}-z_{n}\right)^{2}}\right)-\frac{\delta_{i}^{m} \delta_{j}^{n}}{\left(z_{i}-z_{j}\right)^{2}}
\end{aligned}
$$

which coincides with eq. (3.16) if we take $\widehat{\tau}_{i j}^{a d j}$ there. The simplest of these quantities is

$$
K_{0}(\beta)=\left.\frac{\partial^{2}}{\partial \alpha_{1} \partial \alpha_{2}} K_{\alpha_{1} \alpha_{2}}(\beta)\right|_{\alpha_{1}=\alpha_{2}=0}=<\operatorname{tr}[\varphi(0) \varphi(\beta)]>
$$




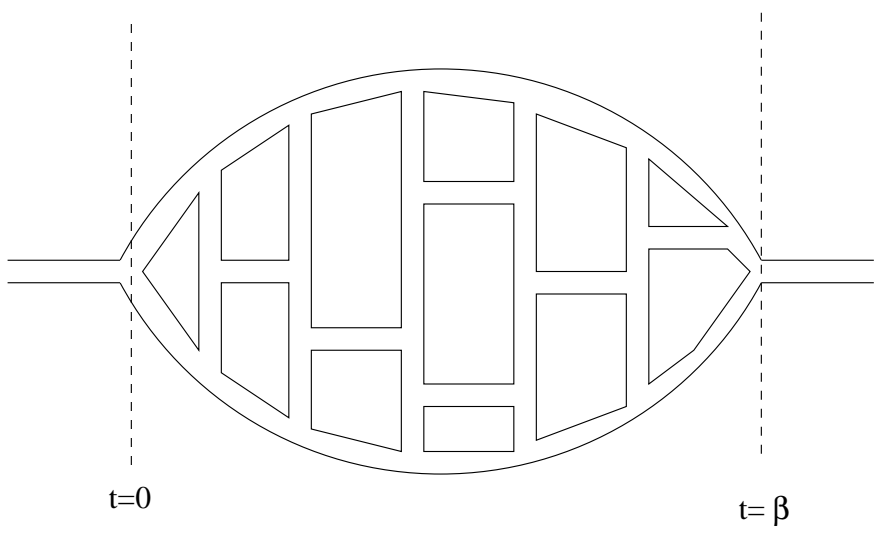

Fig. 2: A typical $\varphi^{3}$ Feynman graph for the adjoint propagator (4.28). It has a topology of a disc with two points on the boundary fixed.

In the case of the matrix $\varphi^{3}$-potential, $K_{0}(\beta)$ will be defined by the diagrams of the type drawn in fig.2. One can see that it represents an open string amplitude with free boundary conditions and two points on the boundary fixed in the t-space at 0 and $\beta$.

On the other hand, this diagrammatic picture allows us to interpret the partition function (4.12) in the adjoint representation directly as describing the one-vortex-antivortex-pair sector of the model.

Indeed, open string is the only intermediate state propagating in the adjoint representation. For $Z_{a d j}$ we have the diagrammatic representation drawn in fig.3. It looks similar to that of the correlator (4.28)) (fig.2) but, instead of having "in" and "out" states at the ends, corresponding to some particular operators $\varphi(0)$ and $\varphi(\beta)$, it is continued periodically looking now as being wrapped around a cylinder. In fact, there are two loops in the graph which wrap around the time circle of the length $\beta$, in opposite directions with respect to each other (if we introduce, say, the clock-wise orientation for every loop). As we mentioned in section 2 it is exactly the definition of vortex and anti-vortex encircled by these two loops, correspondingly. All other loops are small in the $t$-space and do not contain vortices. 


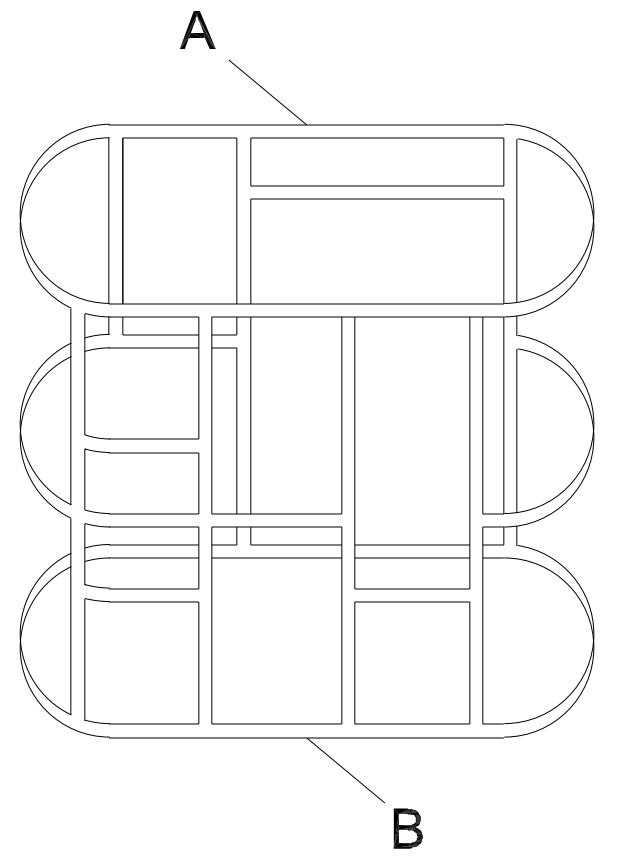

Fig. 3: A typical $\varphi^{3}$ Feynman graph for the adjoint partition function $Z_{\text {adj }}$. It has the topology of a cylinder, which is wrapped around the circle of the radius $\beta / 2 \pi$.

It is possible to generalize this picture to higher representations.

From these considerations we draw the conclusion that higher representations have to be related in some way to open strings with the free (non-renormalized by some additional particles at the ends) boundary conditions and, hence, to bigger numbers of vortices.

Now let us proceed with the partition functions. The untwisted partition function can be defined as:

$$
Z^{(N)}(\beta)=\int d^{N^{2}} \varphi G(\beta, \varphi, \varphi)
$$

Using eqs.(4.16) and (4.29) we can easily reproduce the trivial result (4.2).

The twisted partition function is equal to

$$
Z^{(N)}(\beta, \Omega)=\int d^{N^{2}} \varphi G\left(\beta, \varphi, \Omega^{+} \varphi \Omega\right)
$$

Here we simply have rotated the final states with respect to the initial ones by a matrix $\Omega$. 
Let us first consider the trivial representation (singlet). Using eqs. (4.12) and (4.16) and diagonalizing again the matrix $\varphi$, we integrate over the angular variables by eq.(4.21) and obtain:

$$
\begin{aligned}
Z_{\text {sing }}^{(N)}(\beta)=( & \left.\frac{\omega}{2 \pi \operatorname{sh} \omega \beta}\right)^{N / 2} \int \prod_{k=1}^{N} d z_{k} \exp \left\{-\frac{\omega}{2} \operatorname{cth} \omega \beta z_{k}^{2}\right\} \times \\
& \times \operatorname{det}_{i j}\left[\exp \left\{\frac{\omega}{\operatorname{sh} \omega \beta} z_{i} z_{j}\right\}\right]
\end{aligned}
$$

It is obvious from here that det in eq. (4.31) produces the expected fermionic statistics for $\left\{z_{k}\right\}$. Say, the contribution of configurations with coinciding coordinates $z_{m}=z_{n}$ for some $m$ and $n$, is zero. Later we shall compute $Z_{\text {sing }}$ by other means and reproduce well known results for non-interacting fermions. The measure in eq.(4.30) is invariant under the group transformations $\varphi \rightarrow \omega^{+} \varphi \omega$. We can use this invariance to show that $Z^{(N)}(\beta, \Omega)$ depends only on the eigenvalues of $\Omega$. Indeed, we can use the $\omega$-transformation of $\varphi$ to diagonalize $\Omega$, since nothing depends on $\omega$ :

$$
\left(\omega \Omega \omega^{+}\right)_{k j}=\delta_{k j} e^{i \theta_{j}}
$$

Let us perform now the gaussian integration over $\varphi$ in eq.(4.30). We obtain the useful formula: $\dagger$

$$
Z^{(N)}(\beta, \theta)=q^{\frac{N^{2}}{2}} \prod_{k, m=1}^{N} \frac{1}{1-q e^{i\left(\theta_{k}-\theta_{m}\right)}}
$$

From eqs. (4.12), (4.30) and (4.33) we obtain:

$$
Z_{R}(\beta)=\frac{1}{N !} \int_{0}^{2 \pi} \prod_{k=1}^{N} \frac{d \theta_{k}}{2 \pi}\left|\Delta\left(e^{i \theta}\right)\right|^{2} \chi_{R}\left(e^{i \theta}\right) \prod_{k, m=1}^{N}\left[\frac{e^{-\frac{\omega \beta}{2}}}{1-\exp \left[-\omega \beta+i\left(\theta_{k}-\theta_{m}\right)\right]}\right]
$$

where

$$
\Delta\left(e^{i \theta}\right)=\prod_{k>m}\left(e^{i \theta_{k}}-e^{i \theta_{m}}\right)
$$

and the character $\chi_{R}$ is given by the Weyl formula:

$$
\chi_{R}\left(e^{i \theta}\right)=\frac{\operatorname{det}_{k m}\left(e^{i l_{k} \theta_{m}}\right)}{\Delta\left(e^{i \theta}\right)}
$$

$\dagger$ We thank M. Douglas who showed us this formula. He claimed it was known long time ago but we did not find any references. 
Using the complex variables

$$
\xi_{k}=e^{i \theta_{k}}
$$

eq. (4.34) can be written in the form of the contour integrals:

$$
\begin{aligned}
Z_{R}(q) & =\frac{q^{N^{2} / 2}}{N !} \oint \cdots \oint \prod_{k=1}^{N} \frac{d \xi_{k}}{\xi_{k}}|\Delta(\xi)|^{2} \chi_{R}(\xi) \times \\
& \times \prod_{m, k=1}^{N}\left(1-q \xi_{m} \bar{\xi}_{k}\right)^{-1}
\end{aligned}
$$

The integrals are taken over the unit circle, and all the poles inside this circle are concentrated either at the origin or on the circle of the radius $q$.

In principle, we can obtain by this formula using the direct computation some results for given R and N. However, we need the general formula for arbitrary N.

Let us work out a more effective method for it.

Using the "generating function for characters" [17]:

$$
\prod_{k, l=1}^{N} \frac{1}{1-\xi_{k} \eta_{l}}=\sum_{R} \chi_{R}(\xi) \chi_{R}(\eta)
$$

and the fact that the character is a homogeneous function:

$$
\chi_{R}(p \xi)=p^{\sum_{k=1}^{N} m_{k}} \chi_{R}(\xi)
$$

where $\{m\}$ are again the highest weight components, we can represent eq. (4.34) as:

$$
Z_{R}^{(N)}(q)=\sum_{\left\{R^{\prime}=\ell_{1} \cdots \ell_{N}\right\}} M_{R \otimes R^{\prime}}^{R^{\prime}} \quad q^{\sum_{k=1}^{N}\left(\ell_{k}+\frac{1}{2}\right)}
$$

where the integer numbers $\left\{\ell_{k}\right\}$ obeying the condition

$$
\ell_{1}>\ell_{2} \cdots>\ell_{N}
$$

are related to the components $\left\{n_{k}\right\}$ of the highest weight of $R^{\prime}$ by the formula

$$
\ell_{k}=n_{k}+N-k
$$

and

$$
M_{R \otimes R^{\prime}}^{R^{\prime \prime}}=\frac{1}{N !} \int_{0}^{2 \pi} \prod_{k=1}^{N} \frac{d \theta_{k}}{2 \pi}\left|\Delta\left(e^{i \theta}\right)\right|^{2} \chi_{R}\left(e^{i \theta}\right) \chi_{R^{\prime}}\left(e^{i \theta}\right) \bar{\chi}_{R^{\prime \prime}}\left(e^{i \theta}\right)
$$


is the multiplicity of a representation $R^{\prime \prime}$ in the tensor product $R \otimes R^{\prime}$ of irreducible representations $R$ and $R^{\prime}$. For our purposes we need only the case $R^{\prime}=R^{\prime \prime}$. It is important to note that only non-negative values of $\ell_{k}$ appear in eqs. (4.41), which makes the sum convergent. For the trivial (singlet) representation, we have $\chi_{\text {sing }}\left(e^{i \theta}\right)=1$, and eqs.(4.41)(4.44) give:

$$
\begin{aligned}
Z_{\text {sing }}^{(N)}(q) & =\sum_{\ell_{N}=0}^{\infty} \sum_{\ell_{N-1}=\ell_{N}+1}^{\infty} \cdots \sum_{\ell_{1}=\ell_{2}+1}^{\infty} q^{\sum_{k=1}^{N}\left(\ell_{k}+\frac{1}{2}\right)}= \\
& =\frac{q^{N^{2} / 2}}{(1-q)\left(1-q^{2}\right) \ldots\left(1-q^{N}\right)}
\end{aligned}
$$

We have obtained the partition function of $\mathrm{N}$ fermions in the harmonic potential, as it was expected. The fermionic statistics appears here because of the ordering condition (4.42) for the components of the highest weight (see ref. [18] for some analogies).

The formula (4.41) gives another check of the selection rule mentioned in the section 3: $Z_{R}$ is nonzero only for representations $R=\left\{m_{k}\right\}$ with the sum of highest weight components equal to zero (eq.(3.14)).

If the conditions

$$
n_{k}-n_{k+1} \geq \sum_{j=1}^{N}\left(m_{j}+\left|m_{N}\right|\right)=N\left|m_{N}\right|
$$

(where $\left\{n_{k}\right\}$ are the components of the highest weight of $R^{\prime}$ in eq. (4.41)) are fulfilled for all $1 \leq k<N$, then [17]:

$$
M_{R \otimes R^{\prime}}^{R^{\prime}}=d_{R}^{(0)}
$$

where $d_{R}^{(0)}$ is the dimension of the subspace of all zero weight vectors in the space of a representation $R$ defined by eq. (A6) and in general

$$
M_{R \otimes R^{\prime}}^{R^{\prime}} \leq d_{R}^{(0)}
$$

Eq. (4.47) means that, for $\beta \rightarrow 0$ (or $q=e^{-\omega \beta} \rightarrow 1$ ),

$$
Z_{R}(\beta) \underset{\beta \rightarrow 0}{\sim} \frac{d_{R}^{(0)}}{(\omega \beta)^{N}}
$$

The general structure of the partition function $Z_{R}^{(N)}(q)$ is such that

$$
Z_{R}^{(N)}(q)=P_{R}^{(N)}(q) Z_{\text {sing }}^{(N)}(q)
$$


where $Z_{\text {sing }}^{(N)}(q)$ is the singlet partition function defined by eq. (4.45) and $P_{R}^{(N)}(q)$ is a polynomial in $q$ of a degree not higher than $N \cdot m$, where $m$ is the sum of positive (or negative) components of the highest weight of $R$.

From eq. (4.49) we have:

$$
P_{R}^{(N)}(1)=d_{R}^{(0)}
$$

$P_{R}^{(N)}(q)$ can be regarded as the $q$-analog of the multiplicity of the zero weight and is a known object in modern group theory $\dagger$. They appear to be a particular case of the KostkaGreen-Foulkes polynomials [19]. We elaborated an effective recurrence algorithm suitable for us (see Appendix C for details) which enabled us to obtain for two infinite series of Young tables

$$
A_{n}=(n, 0, \ldots \ldots,-1,-1, \ldots,-1)
$$

and

$$
B_{n}=(n, 0, \ldots \ldots, 0,-n)
$$

the following simple answers for the corresponding generating functions

$$
\begin{aligned}
& \mathcal{P}_{A}^{(N)}(x, q)=\sum_{n=0}^{\infty} P_{A_{n}}^{(N)}(q) x^{n}=\prod_{k=1}^{N-1}\left(1+x q^{k}\right) \\
& \mathcal{P}_{B}^{(N)}(x, q)=\sum_{n=0}^{\infty} P_{B_{n}}^{(N)}(q) x^{n}=\prod_{k=1}^{N-1} \frac{1}{1-x q^{k}}
\end{aligned}
$$

Eqs. (4.54) and (4.55) have the form of the grand canonical partition functions for fermions and bosons, respectively, in the $N-1$ level system where $x$ plays the role of the fugacity. Unfortunately, answers for other representations cannot be represented in a similar simple form but, nevertheless, one can obtain for representations having a small number of nonzero components of their highest weights formulae valid for arbitrary $N$.

\section{Partition functions for the upside-down matrix oscillator}

Now let us try to generalize the methods of the previous section to a more complicated case of the upside down oscillator.

The major complification is the necessity to introduce a cut-off in order to stabilize this unstable system. Any cut-off decreases the high symmetry of the oscillator and makes

$\dagger$ We are indebted to A.Kirillov for drawing our attention to this fact. 
an exact solution impossible. The only tool in our hands is the analytical continuation in the frequency but we should be ready to confront with divergences and, hence, we need additional guesses in order to get physically reasonable answers.

In order to classify the partition functions with respect to $\mathrm{U}(\mathrm{N})$ representations, we have to define again the twisted partition function. As it was noted in the section 4 , we can formally obtain it changing $\omega \rightarrow i \omega$ in the Green function $G\left(\beta, \varphi, \varphi^{\prime}\right)$. Then, after the Gaussian integration over $\varphi$ in eq. (4.30), we formally get the twisted partition function for the upside-down oscillator

$$
Z^{(N)}(\beta, \theta)=e^{-i \omega \beta \frac{N^{2}}{2}} \prod_{k, m=1}^{N}\left[1-e^{-i \omega \beta+i\left(\theta_{k}-\theta_{m}\right)}\right]^{-1}
$$

Instead of trying to give a meaning to eq.(5.1), let us make an attempt to obtain $Z^{(N)}(\beta, \theta)$ solving directly the wave equation

$$
\widehat{H} \Psi(\varphi)=\epsilon \Psi(\varphi)
$$

for $\widehat{H}$ taken from eq. (3.22).

If we forget for a while about the boundary conditions (i.e. the cut-off) we can consider eq. (5.2) as a collection of independent wave equations for off-diagonal matrix elements:

$$
\begin{gathered}
-\frac{1}{2}\left(\frac{\partial^{2}}{\partial \varphi_{i j} \partial \varphi_{i j}^{*}}+\omega^{2} \varphi_{i j} \varphi_{i j}^{*}\right) \Psi_{i j}\left(\varphi_{i j}\right)=\epsilon_{i j} \Psi_{i j}\left(\varphi_{i j}\right) \\
\text { for } \quad i<j
\end{gathered}
$$

and diagonal matrix elements:

$$
\begin{gathered}
-\frac{1}{2}\left(\frac{\partial^{2}}{\partial \varphi_{i i}^{2}}+\omega^{2} \varphi_{i i}^{2}\right) \Psi_{i i}\left(\varphi_{i i}\right)=\epsilon_{i i} \Psi_{i i}\left(\varphi_{i i}\right) \\
\text { for } \quad i=1,2 \cdots N
\end{gathered}
$$

If we introduce the parameterization (dropping for a while the indices $(i, j))$ :

$$
\varphi=\sqrt{r} e^{i \theta}
$$

we get the following form of eq. (5.3):

$$
\chi_{m}^{\prime \prime}+\frac{2}{r} \chi_{m}^{\prime}+\left[\left(\frac{\omega}{2}\right)^{2}+\frac{\epsilon}{2 r}-\frac{m^{2}-1}{4 r^{2}}\right] \chi=0
$$


where $\chi$ is related to $\Psi$ as

$$
\Psi(r, \theta)=\sqrt{r} \sum_{m=-\infty}^{\infty} \chi_{m}(r) e^{i m \theta}
$$

Eq.(5.6) coincides with the Schrödinger equation for the hydrogen atom with the energy $\left(\frac{\omega}{2}\right)^{2}$, the charge $\epsilon / 2$ and the angular momentum $\ell=\frac{|m|-1}{2}$. The imaginary values of $\omega$ in eqs. (5.3) and (5.4) correspond to the discrete spectrum of the hydrogen (negative energies), and we get:

$$
\epsilon=|\omega|(|m|+1+2 k), \quad k=0,1,2 \cdots
$$

The case of real $\omega$ in eq.(5.6) (the upside-down oscillator) corresponds to the continuous spectrum of the hydrogen, and we have to introduce some cut-off to define it.

Again, as in the case of the infinite time interval [6], we have strong reasons to believe that, for the exact definition of the spectrum, only the quasi-classical asymptotic of the wave function suffices:

$$
\begin{gathered}
\chi_{m}(r) \sim \frac{2}{r} \sin \left(r+\frac{\epsilon}{2 \omega} \log (2 r)-\frac{\pi}{2} \frac{|m|-1}{2}+\arg \Gamma\left(\frac{|m|+1}{2}+\frac{i \epsilon}{2 \omega}\right)\right) \\
\text { for } r \gg \frac{1}{2 \omega}\left(m^{2}+\frac{\epsilon^{2}}{\omega^{2}}\right)
\end{gathered}
$$

The Schrödinger equation (5.4) for the diagonal elements describes for an imaginary $\omega$ a collection of standard oscillators having the equidistant spectrum:

$$
\epsilon_{n}=|\omega|\left(n+\frac{1}{2}\right), \quad n=0,1,2 \cdots
$$

and for a real $\omega$ the solution of (5.4) is a parabolic cylinder function having the quasiclassical asymptotics

$$
\begin{gathered}
\Psi(x) \sim x^{-\frac{1}{2}} \exp \left\{ \pm i\left[x^{2}+\frac{\epsilon}{\omega} \log x+\right.\right. \\
\left.\left.+\arg \Gamma\left(i \frac{\epsilon}{\omega}+\frac{1}{2}\right)\right]\right\}
\end{gathered}
$$

valid for $x \gg \frac{\epsilon^{2}}{\omega^{3}}$.

The problem now is that we are, in principle, not allowed to define the energy spectrum for every matrix element, $\varphi_{i j}=\sqrt{r_{i j}} \exp i \theta_{i j}$ and $\varphi_{i i}=x$, separately, since we have the $\mathrm{U}(\mathrm{N})$ invariant boundary condition, say:

$$
\Psi(\varphi)=0 \text { for } \operatorname{tr} \varphi^{2} \geq \Lambda^{2} \sim N
$$


which mixes all matrix elements.

Strictly speaking, to solve this problem we have to consider first the whole wave function for all matrix elements, in the form

$$
\Psi(\varphi)=\sum_{\{\epsilon\}\{m\}} C(\{\epsilon\}) \prod_{i>j} \Psi_{i j}\left(\varphi_{i j}, \epsilon_{i j}\right) \prod_{k} \Psi_{k k}\left(\varphi_{k k}, \epsilon_{k k}\right)
$$

and than find the coefficients $C(\{\epsilon\})$ from the condition (5.13). But we are not able to find them directly.

Instead of doing this we shall assume as in ref. [1] that the essential part of the spectrum is defined by the phase of reflection from the quadratic potential (last terms in the phases of the asymptotics (5.9) and (5.12) ) and not by the infinite wall, appearing in the conditions (5.13).

To justify it let us note that every term in the expansion (5.11) has the quasi-classical asymptotics of the form

$$
\begin{aligned}
& \exp \pm i\left[\operatorname{tr} \varphi^{2}+\frac{1}{\omega} \sum_{i \geq j} \epsilon_{i j} \log \varphi_{i j}+\right. \\
& \left.+\sum_{i>j} \arg \Gamma\left(\frac{\left|m_{i j}\right|+1}{2}+i \frac{\epsilon_{i j}}{2 \omega}\right)+\sum_{k} \arg \Gamma\left(i \frac{\epsilon_{k k}}{\omega}+\frac{1}{2}\right)\right]
\end{aligned}
$$

The first overall term in the phase of eq. (5.15), $e^{ \pm i \operatorname{tr} \varphi^{2}}$ does not have any influence on the density of states, since it is invariant under $\mathrm{U}(\mathrm{N})$ transformations, $\varphi \rightarrow \Omega^{+} \varphi \Omega$, as well as the boundary condition (5.13).

The second logarithmic term, as we hope, will give only slow dependence on the cutoff $\Lambda$ in the density of states, as it was in the simpler situation for the eigenvalues of the matrix $\varphi$ in the singlet representation [4-7].

In other words, we expect that the density of spectrum for every matrix element can be defined from the phase quantization condition. Hence, from (5.9) we have for the off-diagonal degrees of freedom:

$$
\begin{gathered}
\frac{\Lambda^{2}}{2}+\frac{\epsilon}{2 \omega} \log \Lambda^{2}-\frac{\pi}{2} \frac{|m|-1}{2}+\arg \Gamma\left(\frac{|m|+1}{2}+\frac{i \epsilon}{2 \omega}\right)=\pi n \\
n=0,1,2 \cdots
\end{gathered}
$$

which gives for the density of states $\rho_{o}(\epsilon)=\frac{\partial n}{\partial \epsilon}$

$$
\rho_{o}^{(m)}(\epsilon)=-\frac{1}{2 \pi \omega} \operatorname{Re} \psi\left(i \frac{\epsilon}{2 \omega}+\frac{|m|+1}{2}\right)+\frac{1}{2 \pi \omega} \log \Lambda^{2}
$$


where

$$
\psi(x)=-\sum_{k=0}^{\infty}\left(\frac{1}{x+k}-\frac{1}{k+1}\right)-C
$$

is the $\psi$-function of Euler; $C$ is the Euler constant.

For the diagonal degrees of freedom we obtain:

$$
\begin{aligned}
\Lambda^{2}+\frac{\epsilon}{\omega} \log \Lambda^{2}+\arg \Gamma\left(i \frac{\epsilon}{\omega}+\frac{1}{2}\right) & =\pi n \\
n & =0,1,2 \cdots
\end{aligned}
$$

or, for the density of states $\rho_{d}(\epsilon)=\frac{\partial n}{\partial \epsilon}$ :

$$
\rho_{d}(\epsilon)=-\frac{1}{\pi \omega} \operatorname{Re} \psi\left(i \frac{\epsilon}{\omega}+\frac{1}{2}\right)+\frac{1}{\pi \omega} \log \Lambda^{2}
$$

Let us now calculate the twisted partition function for the upside-down matrix oscillator. We shall start from the "twisted density of states" for the off-diagonal matrix elements.

Since $\varphi_{i j}=\sqrt{r_{i j}} e^{i \theta_{i j}}$, the twist $\varphi \rightarrow \Omega^{+} \varphi \Omega$ results in

$$
\theta_{i j} \rightarrow \theta_{i j}+\theta_{i}-\theta_{j}
$$

and we conclude from eqs. (5.7) and (5.17), that

$$
\begin{aligned}
& \rho(\theta, \epsilon)=\sum_{m=-\infty}^{\infty} e^{i m \theta} \rho^{(m)}(\epsilon) \\
& =\frac{1}{\pi} \sum_{k=0}^{\infty} \sum_{m=-\infty}^{\infty} \frac{e^{i m \theta}\left(\frac{|m|+1}{2}+k\right)}{\left(\frac{\epsilon}{\omega}\right)^{2}+\left(k+\frac{|m|+1}{2}\right)^{2}}+\delta(\theta) \log \tilde{\Lambda}^{2}
\end{aligned}
$$

The cut-offs which we have introduced till now are not $\mathrm{U}(\mathrm{N})$ invariant. Therefore, we shall drop in what follows the last term with the periodic $\delta$-function having in mind that the logarithmic divergency will appear on the next stages of the calculations and we will have to recover a cut-off again.

The remaining double sum can be easily calculated (taking $j=|m|+1+2 k$ as a new variable) and the result is:

$$
\rho(\theta, \epsilon)=\frac{\sinh \frac{\epsilon}{\omega}(\pi-\theta)}{\sinh \frac{\epsilon}{\omega} \pi \sin \theta}
$$

It is remarkable that the twisted density of states does not contain a cut-off parameter for $\theta \neq 2 \pi m, m=0, \pm 1, \pm 2 \ldots$. The twisted partition function of a non-diagonal matrix 
element can be calculated taking both parts of the spectrum above and below the top of the potential, i.e. for positive as well as for negative $\epsilon$ :

$$
\begin{aligned}
& Z_{0}(\theta, \beta)=\int_{-\infty}^{+\infty} d \epsilon \mathrm{e}^{-\beta \epsilon} \rho(\theta, \epsilon)= \\
& =\frac{1}{\sin \theta} \int_{-\infty}^{+\infty} d \epsilon \mathrm{e}^{-\beta \epsilon} \frac{\sinh \frac{\epsilon}{\omega}(\pi-\theta)}{\sinh \frac{\epsilon}{\omega} \pi}
\end{aligned}
$$

For

$$
0<\theta<2 \pi
$$

the integral in (5.24) is convergent. The calculation gives:

$$
Z_{0}(\theta, \beta)=\frac{1 / 2}{\cos \beta \omega-\cos \theta}
$$

For $\theta=0$ and $2 \pi$ we understand eq. (5.26) as an analytical continuation of eq. (5.24). If we rewrite eq. (5.26) as

$$
\frac{1 / 2}{\cos \beta \omega-\cos \theta}=\frac{\mathrm{e}^{-i \beta \omega}}{\left(1-\mathrm{e}^{-i(\beta \omega+\theta)}\right)\left(1-\mathrm{e}^{-i(\beta \omega-\theta)}\right)}
$$

we find that this is quite similar to the factor $\frac{1}{1-q e^{i\left(\theta_{k}-\theta_{m}\right)}}$ in eq.(4.33): the differences is only in the change $\omega \rightarrow i \omega$. It means that we do not need to introduce a cut-off for the off-diagonal matrix elements. For the diagonal matrix elements $\varphi_{i i}$, the partition function does not depend on twist angles but the problem is that the integral over energies

$$
Z_{d}(\beta)=\int_{-\infty}^{\infty} d \epsilon e^{-\beta \epsilon} \rho_{d}(\epsilon)=-\int_{-\infty}^{\infty} d \epsilon e^{-\beta \epsilon} \frac{1}{\pi} \operatorname{Re} \psi\left(i \frac{\epsilon}{\omega}+\frac{1}{2}\right)
$$

is highly divergent and needs a cut-off procedure. However, we can formally deform the contour of integration from the real axis to the one shown in fig. 4 and expand $\psi$-function in pole terms (eq.(5.18)). Then we can calculate the integral in eq. (5.28) term by term:

$$
\begin{aligned}
Z_{d}(\beta) & =\frac{1}{\pi} \sum_{h=0}^{\infty} \int d \epsilon e^{-\beta \epsilon} \frac{n+\frac{1}{2}}{\frac{\epsilon^{2}}{\omega^{2}}+\left(n+\frac{1}{2}\right)^{2}}=\sum_{n=0}^{\infty} e^{i \omega \beta\left(n+\frac{1}{2}\right)}= \\
& =\frac{1}{2 \sin \beta \omega / 2}
\end{aligned}
$$




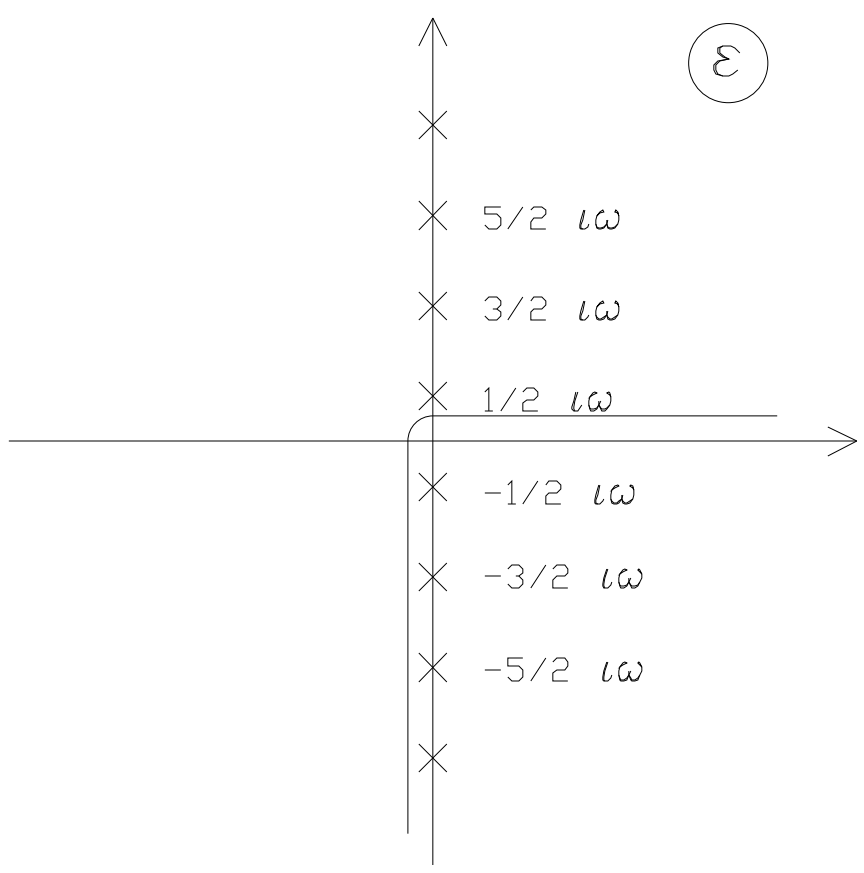

Fig. 4:The contour of integration for the diagonal matrix elements in the partition function (5.28).

If we keep assuming that all $N^{2}$ degrees of freedom $\varphi_{i j}$ for the upside down matrix oscillator are independent, we can write down the whole twisted partition function as a product of $N^{2}$ factors (5.26) and (5.29)

$$
Z^{(N)}(\theta, \beta)=\left(2 \sin \frac{\omega \beta}{2}\right)^{-N} \prod_{i>j} \frac{1}{\cos \omega \beta-\cos \left(\theta_{i}-\theta_{j}\right)}
$$

which coincides with eq.(5.1), and the partition function in a given representation $\mathrm{R}$ takes the form:

$$
Z_{R}^{(N)}(\beta)=\frac{1}{N !} \int_{0}^{2 \pi} \prod_{k=1}^{N} \frac{d \theta_{k}}{2 \pi}\left|\Delta\left(e^{i \theta}\right)\right|^{2} \chi_{R}\left(e^{i \theta}\right) Z^{(N)}(\theta, \beta)
$$

It looks exactly the same as eq.(4.34) for the standard oscillator but with the change $\omega \rightarrow i \omega$. This is an encouraging analogy for us if we remember that we used for the derivation of eq. (5.30) the true spectrum of the upside-down matrix oscillator.

Let us also note that in eq. (5.31) there is no factorization to $N^{2}$ independent degrees of freedom. Effectively, they interact and after integration over the angles only $\mathrm{N}$ effective degrees of freedom remain (e.g. $N$ fermions in the singlet sector). 
Formaly, we can again write eq. (5.31) in the same form as eq. (4.41):

$$
Z_{R}^{(N)}(\beta)=\sum_{S} M_{R \otimes S}^{S} e^{-i \beta \omega \sum_{\kappa=1}^{N}\left(\ell_{\kappa}+\frac{1}{2}\right)}
$$

with the multiplicity $M_{R \otimes S}^{S}$ given by eq.(4.44). But one has now to give a meaning to the imaginary energy levels.

For this purpose let us analyze the simplest case of the singlet representation for which

$$
M_{R \otimes S}^{S}=1
$$

Hence,

$$
\begin{aligned}
Z_{\text {sing }}^{(N)}(\beta) & =\sum_{\left\{\ell_{\kappa}>\ell_{\kappa+1}\right\}} e^{-i \beta \omega \sum_{\kappa=1}^{N}\left(\ell_{\kappa}+\frac{1}{2}\right)}= \\
& =\frac{e^{-i \beta \omega \frac{N^{2}}{2}}}{\left(1-e^{-i \beta \omega}\right)\left(1-e^{-2 i \beta \omega}\right) \cdots\left(1-e^{-N i \beta \omega}\right)}
\end{aligned}
$$

which can be reproduced from the result for the singlet representation (4.45) of the standard oscillator by change $\omega \rightarrow i \omega$.

How to give a meaning to eq.(5.34)? Let us pass from the microcanonical to the grand canonical ensemble. The singlet partition function takes the form

$$
\begin{aligned}
& Z_{\text {sing }}(\mu, \beta)=\sum_{N=0}^{\infty} Z^{(N)}(\beta) e^{\beta \mu}= \\
& =\exp \sum_{k=0}^{\infty} \log \left(1+e^{\beta\left[\mu-i \omega\left(k+\frac{1}{2}\right)\right]}\right)
\end{aligned}
$$

in the complete analogy with the well-known expression for the grand canonical partition function of fermions in the oscillatorial potential. As usually, the complex energy levels have to be understood as resonances [1,4-7]:

$$
\begin{aligned}
f_{\text {sing }}(\mu, \beta) & =\log Z_{q \operatorname{sing}}(\mu, \beta)=\int_{-\infty}^{+\infty} d \epsilon \frac{1}{\pi} \sum_{k=0}^{\infty} \frac{k+\frac{1}{2}}{\left(\frac{\epsilon}{\omega}\right)^{2}+\left(k+\frac{1}{2}\right)^{2}} \log \left[1+e^{\beta(\mu-\epsilon)}\right] \\
& =\int_{-\infty}^{\infty} d \epsilon \rho_{\text {sing }}(\epsilon) \log (1+\exp \beta(\mu-\epsilon))
\end{aligned}
$$

where $\rho_{\text {sing }}(\epsilon)$ coincides with eq.(5.20). 
It is assumed that every integral in the sum is to be calculated as a pole contribution of the denominator in eq. (5.36) ignoring the singularities of $l o g$. In fact, at the lower limit, the integral in eq. (5.36) is logarithmically divergent and one has to cut the integration by some minimal energy $\epsilon_{0}\left(\left|\epsilon_{0}\right| \sim N\right)$, which is defined by the bottom of the original potential. Furthermore, the sum over resonances also diverges and needs to be regularized as well. Nevertheless, we have reproduced the correct result representing $f_{\text {sing }}$ as the partition function of $N$ fermions in the upside-down oscillatorial potential [2]. Now, assuming that the general structure of answers is the same for all representations, we can proceed and get for the adjoint using eq. (C10) the following formal expression:

$$
Z_{\text {adj }}^{(N)}(\beta)=\frac{e^{-\frac{3}{2} i \omega \beta}}{1-e^{-i \omega \beta}} e^{-i \omega \beta(N-1)} Z_{\text {sing }}^{(N-1)}(\beta)-e^{-i \omega \beta N} Z_{\operatorname{sing}}^{(N)}(\beta)
$$

and, in the grand canonical form,

$$
\begin{aligned}
& Z_{\mathrm{adj}}(\mu, \beta)=\sum_{N=0}^{\infty} e^{\beta \mu N} d_{a d j} Z_{\mathrm{adj}}^{(N)}(\beta)= \\
& =\left(\left(\frac{1}{\beta} \frac{\partial}{\partial \mu}\right)^{2}-1\right)\left(A(\mu, \beta) Z_{\mathrm{sing}}(\mu-i \omega, \beta)+B(\mu, \beta)\right)
\end{aligned}
$$

where we have represented the dimension of the adjoint,$d_{a d j}=N^{2}-1$, as the differential operator and pulled it out of the sum over $N$.

$$
A(\mu, \beta)=\frac{\mathrm{e}^{\beta\left(\mu-\frac{3}{2} i \omega\right)}}{1-\mathrm{e}^{-i \beta \omega}}-1
$$

If we define that

$$
Z_{\mathrm{adj}}^{(0)}(\beta)=Z_{\mathrm{sing}}^{(0)}(\beta) \lim _{N \rightarrow 0} \frac{e^{i \omega \beta}-e^{-i \omega \beta N}}{1-e^{-i \omega \beta}}=-1
$$

then

$$
B(\mu, \beta)=0
$$

In any case, this constant is determined by the first terms in the grand canonical expansion in eq. (5.38) and cannot influence universal properties of $Z_{\text {adj }}(\mu, \beta)$.

Now using the same procedure which has led us from eq. (5.35) to eq. (5.36) we obtain:

$$
\begin{aligned}
& Z_{\mathrm{adj}}(\mu, \beta)=\left(\left(\frac{1}{\beta} \frac{\partial}{\partial \mu}\right)^{2}-1\right) A(\mu, \beta) Z_{\mathrm{sing}}(\mu-i \omega, \beta)= \\
& =\left(\left(\frac{1}{\beta} \frac{\partial}{\partial \mu}\right)^{2}-1\right) A(\mu, \beta) \exp \sum_{k=1}^{\infty} \int_{-\infty}^{+\infty} \frac{d \epsilon}{\pi \omega} \frac{k+\frac{1}{2}}{\left(\frac{\epsilon}{\omega}\right)^{2}+\left(k+\frac{1}{2}\right)^{2}} \log \left(1+e^{\beta(\mu-\epsilon)}\right) \\
& =\left(\left(\frac{1}{\beta} \frac{\partial}{\partial \mu}\right)^{2}-1\right) A(\mu, \beta) \exp \int_{-\infty}^{\infty} d \epsilon \rho_{\mathrm{adj}}(\epsilon) \log \left(1+e^{\beta(\mu-\epsilon)}\right)
\end{aligned}
$$


where

$$
\rho_{\text {adj }}(\epsilon)=-\frac{1}{\pi \omega} \operatorname{Re} \psi\left(i \frac{\epsilon}{\omega}+\frac{3}{2}\right)+\frac{1}{\pi \omega} \log \Lambda
$$

or

$$
\rho_{\text {adj }}(\epsilon)=\rho_{\text {sing }}(\epsilon)-\frac{1}{2 \pi \omega} \frac{1}{\left(\frac{\epsilon}{\omega}\right)^{2}+\frac{1}{4}}
$$

We see that the effective density of states in the adjoint representation differs from the singlet one by one missing Lorentzian (resonance).

The factor $A(\mu, \beta)$ could be rewritten with the same argumentation in the form

$$
A(\mu, \beta)=\int_{-\Lambda^{2}}^{\infty} d \epsilon \rho_{\text {adj }}(\epsilon) e^{\beta(\mu-\epsilon)}-1
$$

and interpreted as the partition function of one particle decoupled from the others, even though we do not have any microscopic arguments supporting this interpretation. Nevertheless, it seems to give just a non-universal factor of the order $\exp \left(\beta \Lambda^{2}\right)$ which does not affect universal behavior. Of course, this particle does interact with others at distances of the order $\Lambda$, but, being taken into account, this interaction produces only exponentially small in $N$ terms and does not change the double scaling limit.

Unfortunately, except for the adjoint representation, the procedure of the analytical continuation is ambiguous. If we collect together four representations of the next order (corresponding polynomials given by eqs.(C11), (C12), (C13)) and implement the same procedure as led us to eq. (5.38), we shall get

$$
\begin{aligned}
Z_{2}(\mu, \beta) & =\sum_{N} e^{\beta \mu N}\left(2 d_{A_{2}} Z_{A_{2}}^{(N)}(\beta)+d_{B_{2}} Z_{B_{2}}^{(N)}(\beta)+d_{C_{2}} Z_{C_{2}}^{(N)}(\beta)\right) \\
& =\frac{e^{\beta(2 \mu-2 i \omega)}}{\left(1-e^{-i \omega \beta}\right)\left(1-e^{-2 i \omega \beta}\right)}\left\{\frac{e^{-i \omega \beta}}{2}\left(\left(\frac{1}{\beta} \frac{\partial}{\partial \mu}\right)^{2}-1\right)\left(\left(\frac{1}{\beta} \frac{\partial}{\partial \mu}\right)^{2}-4\right)+\right. \\
& \frac{e^{-2 i \omega \beta}}{4}\left(\frac{1}{\beta} \frac{\partial}{\partial \mu}\right)^{2}\left(\frac{1}{\beta} \frac{\partial}{\partial \mu}-1\right)\left(\frac{1}{\beta} \frac{\partial}{\partial \mu}+3\right)+ \\
& \left.\frac{1}{4}\left(\frac{1}{\beta} \frac{\partial}{\partial \mu}\right)^{2}\left(\frac{1}{\beta} \frac{\partial}{\partial \mu}+1\right)\left(\frac{1}{\beta} \frac{\partial}{\partial \mu}-3\right)\right\} Z_{\operatorname{sing}}(\mu-2 i \omega, \beta) \\
& +\frac{e^{\beta\left(\mu-\frac{1}{2} i \omega\right)}}{\left(1-e^{-i \omega \beta}\right)}\left\{\frac{1}{2}\left(\left(\frac{1}{\beta} \frac{\partial}{\partial \mu}\right)^{2}-1\right)\left(\left(\frac{1}{\beta} \frac{\partial}{\partial \mu}\right)^{2}-4\right)+\right. \\
& \left.\frac{1}{4}\left(\frac{1}{\beta} \frac{\partial}{\partial \mu}\right)^{2}\left(\frac{1}{\beta} \frac{\partial}{\partial \mu}+1\right)\left(\frac{1}{\beta} \frac{\partial}{\partial \mu}-3\right)\right\} Z_{\operatorname{sing}}(\mu-i \omega, \beta) \\
& +\frac{1}{2}\left(\left(\frac{1}{\beta} \frac{\partial}{\partial \mu}\right)^{2}-1\right)\left(\left(\frac{1}{\beta} \frac{\partial}{\partial \mu}\right)^{2}-4\right) Z_{\operatorname{sing}}(\mu, \beta)
\end{aligned}
$$


where we have substituted $d_{A_{2}}=\frac{1}{4}\left(N^{2}-1\right)\left(N^{2}-4\right) ; d_{B_{2}}=\frac{1}{4} N^{2}(N-1)(N+3) ; d_{C_{2}}=$ $\frac{1}{4} N^{2}(N+1)(N-3)$ and converted them into the differential operators.

Eq. (5.46) should contain, as we believe, contributions describing different configurations having the total vortex (anti-vortex) charge equal to 2. Moreover, there should be a contribution from two vortex pairs living on different surfaces (since we deal with the partition function rather than the free energy). In order to perform an analytical continuation, one should presumably separate all those terms and treat them differently.

\section{Physical results in the double scaling limit}

The results for the upside down oscillator given by eqs.(5.36) and (5.42) allow us to obtain the double scaling limit for the compactified one dimensional string (or for the XY-model coupled to gravity) in the sector with one vortex-anti-vortex pair.

The partition function for the singlet representation eq. (5.36) was investigated in ref. [2]. It corresponds to the case of no vortices, when only nontrivial cycles of a twodimensional manifold (given by a Feynman graph with non-trivial topology) can wrap around the time circle of length $\beta$.

\section{Singlet partition function}

Let us repeat the calculation of $f_{\text {sing }}(\beta, \mu)$ from ref. [2] for the consistency of content. We have to remember that the eq.(5.36) describes a filling by free fermions of both sides of the inverted oscillatorial potential. However, in the string perturbation theory which will correspond here to $1 / \mu$-expansion we have to take into account only the states on one side of this potential, which corresponds to the perturbative vacuum of this string theory. This is reflected by an overall factor $1 / 2$ in the following formula for the singlet free energy:

$$
f_{\text {sing }}^{(\text {pert })}(\mu)=f_{\text {sing }}^{r e g}(\mu)+\frac{1}{2} \int_{-\infty}^{\infty} d \epsilon \rho_{\text {sing }}(\epsilon) \log \left(1+e^{\beta(\mu-\epsilon)}\right)
$$

where $f_{\text {sing }}^{\text {reg }}(\mu)=A_{0}(\lambda)+A_{1}(\lambda) \mu+A_{2}(\lambda) \mu^{2}+\cdots$ is the regular part of expansion of the grand canonical free energy in $\mu$. Using an integral representation for $\rho_{\operatorname{sing}}(\epsilon)$

$$
\rho_{\text {sing }}(\epsilon)=\frac{1}{2 \pi} R e \int_{\Lambda^{-1}}^{\infty} d x \frac{e^{i \epsilon x}}{\sinh \frac{x}{2}}
$$

(we put $\omega=1$, fixing appropriately the scale of $\epsilon$ ) and integrating over $\epsilon$ in the eq. (6.1), we obtain the following formula for the free enery of singlet states

$$
\frac{\partial^{3} f_{\text {sing }}}{\partial \mu^{3}}=\frac{\beta}{2 \pi} \operatorname{Im} \int_{0}^{\infty} d x \frac{\frac{x}{2}}{\sinh \frac{x}{2}} \frac{\frac{\pi x}{\beta}}{\sinh \frac{\pi x}{\beta}} e^{i \mu x}
$$


Expanding $f_{\text {sing }}$ in $1 / \mu$ and introducing a cut-off $\Lambda$ to regularize the logarithmic divergences we get:

$$
f^{(\text {sing })}(\mu)=\frac{\beta}{2 \pi}\left[-\frac{1}{2} \mu^{2} \log \left(\mu / \Lambda^{2}\right)-\frac{1}{24}\left(1+\left(\frac{2 \pi}{\beta}\right)^{2}\right) \log \left(\mu / \Lambda^{2}\right)+\sum_{k=2}^{\infty} \mu^{-2(k-1)} f_{k}(\beta)\right]
$$

where we introduced polynomials in $\frac{1}{\beta}$

$$
f_{k}(\beta)=(2 k-3) ! 2^{-2 k} \sum_{n=0}^{k}\left(\frac{2 \pi}{\beta}\right)^{2 n} \frac{\left(2^{2(k-n)}-2\right)\left(2^{2 n}-2\right)\left|B_{2(k-n)}\right|\left|B_{2 n}\right|}{[2(k-n)] ![2 n] !}
$$

and $B_{m}$ are the Bernoulli numbers.

We do not have a direct interpretation of this grand canonical partition function as a sum over world sheets embedded into a time circle and classified by specific topologies. Such interpretation exists only for the canonical ensemble where we fix the variable $N$ instead of $\mu$. The relation between two ensembles is given by the integral transform:

$$
\exp \left[f_{N}^{s i n g}\right]=\oint d \mu \exp \left[-\beta \mu N+f^{\operatorname{sing}}(\mu)\right]
$$

where the integration contour encircles the point $e^{\beta \mu}=0$. The $1 / N$ expansion of $f_{N}^{\text {sing }}(\lambda)$ provides the string partition functions of given genera.

In the double scaling limit which is the continuum limit of the string theory we have to extract only the lading singularity at $\lambda \rightarrow \lambda_{c}$. To do it let us rewrite (6.4) in the form

$$
\exp \left[f_{N}^{\text {sing }}\right]=\oint d \mu \exp \left[\frac{\beta}{2 \pi} \mu \mu_{0} \log \mu_{0}+f^{\text {sing }}(\mu)\right]
$$

where we introduced the parameter $\mu_{0}$ by the formula

$$
\mu_{0} \log \left(\mu_{0} / \Lambda^{2}\right)=2 \pi\left[A_{1}(\lambda)-N\right] \sim N \Delta
$$

with $\Delta=\left(\lambda_{c}^{2}-\lambda^{2}\right) / \lambda_{c}^{2}$.

The integral can be calculated in terms of the series in $1 / \mu_{0}$ which is equivalent to the $1 / N$ expansion. To generate this series we have to apply the saddle point approximation

$1 \quad \lambda_{c}$ is defined in such a way that in the planar limit $N \rightarrow \infty$ when the temperature $\beta^{-1}$ is effectively zero (the spherical string amplitude does not feel the compactness of "time") we have $\mu\left(\lambda_{c}\right)=0$ 
and then to take systematically into account the fluctuations around the saddle point. The saddle point for $\mu\left(\mu_{0}\right)$ can be found from the equation:

$$
\mu_{0} \log \left(\mu_{0} / \Lambda^{2}\right)=\frac{2 \pi}{\beta} \frac{\partial f_{\text {sing }}^{\text {pert }}(\mu)}{\partial \mu}
$$

Solving it iteratively we obtain:

$$
\mu=\mu_{0}\left[1-\frac{1}{\log \left(\mu_{0} / \Lambda^{2}\right)}\left(\sum_{k=1}^{\infty} \mu_{0}^{-2 k} 2(k-1) f_{k}(\beta)\right)+O\left(\frac{1}{\log ^{2}\left(\mu_{0} / \Lambda^{2}\right)}\right)\right]
$$

We see that in the double scaling limit when $\mu_{0}$ is kept finite and $\Lambda \rightarrow \infty$ we can take $\mu=\mu_{0}$ as a saddle point.

Then we have to put $\mu=\mu\left(\mu_{0}\right)+\delta \mu$ and integrate over $\delta \mu$ in the Gaussian approximation. The contribution to $f_{N}^{s i n g}$ from this Gaussian integration looks as $-\frac{1}{2} \log \log \left(\mu_{0} / \Lambda^{2}\right)+O\left(\frac{1}{\log \left(\mu_{0} / \Lambda^{2}\right)}\right)$. These terms should be neglected with the same accuracy.

The net result of this calculation is that with the accuracy up to inverse logarithmic corrections we substitute the integral (6.5) by its saddle point value at $\mu=\mu_{0}$ and get the following result for the canonical free energy of singlet states [2]:

$$
f_{N}^{s i n g}=\frac{\beta}{2 \pi}\left[\frac{1}{2} \mu_{0}^{2} \log \left(\mu_{0} / \Lambda^{2}\right)-\frac{1}{24}\left(1+\left(\frac{2 \pi}{\beta}\right)^{2}\right) \log \left(\mu_{0} / \Lambda^{2}\right)+\sum_{k=2}^{\infty} \mu_{0}^{-2(k-1)} f_{k}(\beta)\right]
$$

Note the change of sign of the first term (spherical free energy) with respect to the similar term in $(6.3)$.

As it was noticed in ref. [2] eqs. (6.2),(6.3) and (6.8) possess a T-duality symmetry with respect to the inversion of the radius of compactification:

$$
\frac{\beta}{2 \pi} \rightarrow \frac{2 \pi}{\beta} ; \quad \mu \rightarrow \frac{\beta}{2 \pi} \mu
$$

This important property remains to be true in every order of the topological $(1 / \mathrm{N})$ expansion (we recall that $\mu \sim N \frac{\Delta}{|\log \Delta|}$ in the planar limit).

\section{Adjoint partition function}


The analytical continuation of the partition function for the adjoint representation (eq. (5.42)) looks ambiguous but nevertheless we assume that its universal part can be written as follows

$$
Z_{a d j}=\left(\frac{1}{\beta^{2}} \frac{\partial^{2}}{\partial \mu^{2}}-1\right) \exp f_{a d j}(\mu, \beta)
$$

where $f_{\text {adj }}(\mu, \beta)$ is determined similarly to $f_{\text {sing }}(\mu, \beta)$ but with the density of states given by eq. $(5.45)$

$$
\rho_{\text {adj }}(\epsilon)=\rho_{\text {sing }}(\epsilon)-\frac{1}{\pi} \frac{1 / 2}{\epsilon^{2}+1 / 4}
$$

Hence, we have

$$
\delta=f_{\text {adj }}-f_{\text {sing }}=\frac{1}{2} \int d \epsilon\left(\rho_{\text {adj }}(\epsilon)-\rho_{\text {sing }}(\epsilon)\right) \log \left(1+e^{\beta(\mu-\epsilon)}\right)
$$

Using the representation

$$
\frac{z}{\epsilon^{2}+z^{2}}=\operatorname{Re} \int_{0}^{\infty} d x e^{i x \epsilon-z x}
$$

and integrating in eq. (6.11) over $\epsilon$ we obtain for the third derivative of $\delta$ the following representation

$$
\frac{\partial \delta}{\partial \mu}=\frac{1}{2} \int_{0}^{\infty} d x \frac{\sin (\mu x) \sinh \frac{x}{2}}{\sinh \frac{\pi x}{\beta}}
$$

And the topological expansion of $\delta$ takes the form

$$
\begin{aligned}
\delta(\mu) & =\frac{\beta}{2 \pi}\left[\log \left(\mu / \Lambda^{2}\right)-\frac{1}{8 \mu^{2}}\left(1-\frac{1}{3}\left(\frac{2 \pi}{\beta}\right)^{2}\right)\right. \\
& \left.-\sum_{m=2}^{\infty} \mu^{-2 m}(-1)^{m}(2 m-1) ! 2^{-2 m} \sum_{n=0}^{m}\left(\frac{2 \pi}{\beta}\right)^{2 n} \frac{\left(2^{2 n}-2\right) B_{2 n}}{[2(m-n)] ! !(2 n) !}\right]
\end{aligned}
$$

From eq. (6.14) we conclude that

$$
f_{\text {adj }}-f_{\text {sing }} \underset{\mu \rightarrow \infty}{\sim} \frac{\beta}{2 \pi} \log \frac{\mu}{\Lambda^{2}}
$$

Since $\mu \sim \Delta \cdot N$ in the first approximation (and $\Lambda^{2} \sim N$ ), we reproduce in eq. (6.15) the result (1.1) from the paper [3]: the spectrum of angular (vortex) excitations is separated from the singlet (vortex-free) spectrum by a logarithmically big gap. Note that the expression (6.14) is not self-T-dual anymore.

This fact can be qualitatively explained from the comparison of the sum-over-surfaces picture for the correlator eq. (4.20) propagating the singlet states and the correlator (4.28) 
propagating the adjoint states. The first one is given by the sum over all Feynman graphs with two vertices fixed (on a graph and in the target space). These two vertices are arbitrary and can belong to any two different loops or the same loop on a graph.

In the case of the correlator (4.28) one has to pick up two vertices belonging to the same loop on a graph, which is only a part of (positively defined) contributions of the correlator (4.20). Hence

$$
\left.\frac{\partial^{2}}{\partial \alpha_{1} \partial \alpha_{2}} D_{\alpha_{1} \alpha_{2}}(\beta)\right|_{\alpha_{1}=\alpha_{2}=0}=D_{0}(\beta)>K_{0}(\beta)
$$

If we take for the definition of the gap the large $\beta$ asymptotics:

$$
\begin{aligned}
& D_{0}(\beta) \sim \exp \left(-m_{\text {sing }} \cdot \beta\right) \\
& K_{0}(\beta) \sim \exp \left(-m_{\text {adj }} \cdot \beta\right)
\end{aligned}
$$

we come from eq. (6.16) to the conclusion that

$$
m_{\text {adj }}>m_{\text {sing }}
$$

Hence, it is not surprising that

$$
m_{\text {sing }} \sim \frac{1}{|\log \Delta|} \underset{\Delta \rightarrow 0}{\rightarrow} 0
$$

and

$$
m_{\mathrm{adj}} \sim|\log \Delta| \underset{\Delta \rightarrow 0}{\rightarrow \rightarrow 0}+\infty
$$

As we demonstrated in the section 4 , higher representations correspond to the multiloop amplitudes of open strings with free boundary conditions on the edges of the world sheet. We have no parameter (like the mass of a particle at the end-points of the open string) to adjust in order renormalize the "boundary tension" and to make the characteristic size of the boundary macroscopic. Eqs. (6.20) and (6.21) show that the characteristic boundaries have very short lengths, and the contribution of few vortices is negligible in comparison with the contributions of vortex-free configurations.

However, the vortices have a considerable entropy, which causes at some $\beta$ the Berezinski-Kosterlitz-Thouless phase transition $[13,14]$. Knowing the partition function for one vortex-anti-vortex pair, we can calculate the critical value, $\beta_{K T}$, as follows. From the eqs. (3.15), (6.8) and (6.15) we obtain in the spherical approximation in the canonical 
(fixed $N$ ensemble) the following partition function of the vortex anti-vortex pair in the $c=1$ string theory $[3]$ :

$$
\begin{aligned}
\mathcal{Z}_{N}(\beta, \lambda) & \simeq Z_{\text {sing }}\left(1+N^{2} e^{f_{a d j}-f_{\text {sing }}}+\cdots\right) \\
& \simeq \exp \left(\frac{\beta}{2 \pi} N^{2} \frac{1}{2} \Delta^{2}\left[\log (\Delta)+\operatorname{const} \Delta^{\frac{\beta}{2 \pi}-2}+\cdots\right]\right)
\end{aligned}
$$

We see that the second term (as well as the contributions with more vortices and antivortices) is irrelevant with respect to the first one, describing the usual scaling of the $c=1$ string with no vortices, when $\beta>4 \pi$. However, it becomes more important than the first term for $\beta<4 \pi$ and the dilute gas approximation for vortex-antivotex pairs used in the eq. (6.22) is not valid any more. The world sheet of the string will be immediately densely populated by the strongly interacting vortex plasma. It is a typical picture of the Berezinski-Kosterlitz-Touless phase transition.

This phase transition occurs in our model at the same inverse temperature

$$
\beta_{K T}=4 \pi
$$

as in the model of planar rotors ( $X Y$ model) on the plane. Indeed, the answer (6.22) should be compared with the configuration integral of the vortex-anti-vortex pair on the plane $[13,14]$ (in the same normalization):

$$
Q=\int d^{2} x \int d^{2} y \exp -\frac{\beta}{2 \pi} \log \frac{|x-y|^{2}}{r_{o}^{2}} \sim V^{2-\frac{\beta}{2 \pi}}
$$

where $V$ is the volume of the space.

\section{A possible approach to $\mathrm{D}+1$-dimensional bosonic string}

A natural generalization of the matrix model describing one-dimensional strings to the case of $\mathrm{D}+1$-dimensional bosonic strings is the following $\mathrm{D}+1$-dimensional scalar matrix field theory:

$$
Z_{N}(\beta, \lambda)=\int \mathcal{D}^{N^{2}} \varphi(x, t) \exp -N \operatorname{tr} \int d t \int d^{D} x\left[\frac{1}{2}\left(\partial^{\mu} \varphi \partial_{\mu} \varphi\right)+V(\varphi)\right]
$$

with the potential $V(\varphi)$ given by eq.(4.3) or of a more general form. 
The analogy with the string theory is again based on the Feynman graphs: we obtain for the free energy $f_{N}^{(D)}=\frac{1}{N^{2}} \log Z_{N}^{(D)}$ the following diagrammatic expansion

$$
f_{N}(\beta)=\sum_{g=0}^{\infty} N^{-2 g} \sum_{k=0}^{\infty} \lambda^{k} \sum_{G_{g}^{(n)}} \int d^{D+1} x_{1} \cdots d^{D+1} x_{n} \prod_{<i j>\in G_{g}^{(n)}} D\left(x_{i}-x_{j}\right)
$$

where

$$
D(x)=\frac{1}{(2 \pi)^{D+1}} \int d^{D+1} p e^{i(p x)} \frac{1}{p^{2}+\omega^{2}}
$$

As long as we have the superconvergibility of the integrals in eq. (7.2) (it is true, say, for $D<D_{c}=5$ for $\varphi^{3}$ graphs) we may hope that the particular microscopic definition of $D(x)$ is irrelevant, and for big enough k's in eq. (7.2) we have defined the $D+1$-dimensional bosonic string field theory.

Unfortunately, the theory (7.1) is much more complicated than the theory (2.1). We cannot, for example, obtain a representation similar to the Hamiltonians (3.16) for the eigenvalues of the matrix field: the corresponding "connection" $A_{\mu}=\Omega^{+} \partial_{\mu} \Omega$ (compare with eq.(3.3)), obeys now the more complicated constraint, $\partial_{\mu} A_{\nu}-\partial_{\nu} A_{\mu}+\left[A_{\mu}, A_{\nu}\right]=0$, and the integration over $A_{\mu}$ is highly nontrivial. The importance of the angular variables for $C=D+1>1$ physics is reflected in the fact that the eigenvalues may be now not the only and even not the most important degrees of freedom.

On the other hand, if we still hope that the physics at $C>1$ bears some universal features, we may admit that the particular shape of the potential $V(\varphi)$ should not be important. All the results for $C<1$ show that only singular points like local maximum or inflection points are essential for the continuum limit of the corresponding string theory, and all the rest in the shape of the potential after an appropriate rescaling serves as a cut-off.

This brings to mind the idea that it may be sufficient to consider the most generic unstable potential:

$$
V(\varphi)=-\frac{m^{2}}{2} \varphi^{2}
$$

Forgetting for a while about the rest of the potential, we can use the solvable Gaussian theory

$$
Z_{N}=\int \mathcal{D}^{N^{2}} \varphi(x) \exp -\frac{N}{2} \operatorname{tr} \int d^{D+1} x\left[\left(\partial_{\mu} \varphi\right)^{2}+m^{2} \varphi^{2}\right]
$$

in order to get the results for the unstable theory (7.4) by means of the analytical continuation

$$
m \rightarrow i m
$$


and an appropriate introduction of a cut-off. If we will be unable to find this procedure so the whole approach based on the matrix model (7.1) does not describe any universal bosonic string theory for $c>1$.

Again as in the case $c=1$, the Gaussian theory (7.5) is too poor to be a starting point for an analytical continuation, and we have to consider the states obeying some particular representation of the $\mathrm{U}(\mathrm{N})$ group (since the potential $\operatorname{tr} V(\varphi)$ is still invariant under $\varphi \rightarrow \Omega^{+} \varphi \Omega$ transformation and so is the cut-off procedure).

It is natural to hope that the ground state of the system is again the singlet under the group transformations; therefore, we have to extract only the singlet part of eq. (7.5) and then to perform the analytical continuation.

In order to find the partition function for the singlet states we introduce again the twisted partition function: we compactify the time dimension on the circle of a length $\beta$ $(t \in(0, \beta)$ again) and for the remaining $D$ directions we use the momentum representation:

$$
\begin{gathered}
Z_{N}^{D}(\beta, \Omega)=\int \mathcal{D}^{N^{2}} \varphi_{p}(t) \exp \frac{N}{2} \operatorname{tr} \int_{0}^{\beta} d t \int d^{D} p\left[\dot{\varphi}^{2}-\left(p^{2}+m^{2}\right) \varphi^{2}\right] \\
\varphi_{p}(0)=\Omega^{+} \varphi_{p}(\beta) \Omega
\end{gathered}
$$

where we have introduced the $\mathrm{U}(\mathrm{N})$ twisted periodic initial conditions.

For fixed p every matrix element can be considered as an independent oscillator with the effective frequency

$$
\omega_{p}=\sqrt{p^{2}+m^{2}}
$$

Hence, in the complete analogy with eqs. (4.33) or (5.30) we can write:

$$
Z_{N}^{(D)}(\beta, \theta)=\exp -\sum_{k \geq j=1}^{N} F\left(\beta, \theta_{k}-\theta_{j}\right)
$$

where

$$
F(\beta, \theta)=L^{D} \int d^{D} p \log \left[\cosh \frac{\beta}{2} \sqrt{p^{2}+m^{2}}-\cos \theta\right]
$$

$\mathrm{L}$ is an infrared cut-off (the size of the box where the system is put in the $\mathrm{D}$ dimensional target space), and the twist matrix $\Omega$ is chosen in the diagonal form

$$
\Omega=\operatorname{diag}\left(e^{i \theta_{1}}, e^{i \theta_{2}}, \cdots e^{i \theta_{N}}\right)
$$


Then the recipe to find the ground state $E_{0}$ of our model is to calculate the singlet partition function:

$$
Z_{\text {sing }}^{(D)}(\beta)=\int_{0}^{2 \pi} \cdots \int_{0}^{2 \pi} \prod_{k=1}^{N} \frac{d \theta_{k}}{2 \pi}\left|\Delta\left(e^{i \theta}\right)\right|^{2} Z_{N}^{(D)}(\beta, \theta)
$$

to perform the analytical continuation (7.6), introducing the cut-off on the way, which should defined the dependence on the cosmological constant, and to find the asymptotics

$$
Z_{\text {sing }}^{(D)}(\beta) \underset{\beta \rightarrow \infty}{\longrightarrow} e^{-\beta E_{0}}
$$

Of course, all these steps do not seem to be trivial. One of many obstacles to overcome is the highly divergent integral in the eq. (7.8). one has to learn how to deal with these divergences. But what is hopeful here is the fact that the integral in eq. (7.10) goes over only $\mathrm{N}$ (and not $N^{2}$ ) variables and is in principle of a saddle point type in the large $\mathrm{N}$ limit.

Another way to proceed is to try to use eq. (4.39) in order to obtain a representation similar to eq. (4.41):

$$
\begin{aligned}
Z_{R}^{(D)}(\beta) & =\int_{0}^{2 \pi} \prod_{k=1}^{N} \frac{d \theta_{k}}{2 \pi}\left|\Delta\left(e^{i \theta}\right)\right|^{2} \chi_{R}\left(e^{i \theta}\right) \prod_{\{p\}} \prod_{k \leq j} \mathrm{e}^{F\left(\beta, \theta_{k}-\theta_{j}\right)} \\
& =\sum_{S_{p}} \mathrm{e}^{-\beta \omega_{p} \sum_{k=1}^{N}\left(\ell_{k, p}+\frac{1}{2}\right)} \int(d U) \chi_{R}(U) \prod_{\{p\}} \chi_{S_{p}}(U) \bar{\chi}_{S_{p}}(U) \\
& =\sum_{S_{p}} M_{R\left[\otimes S_{p}\right]}^{\left[\otimes S_{p}\right]} \mathrm{e}^{-\beta \omega_{p} \sum_{k=1}^{N}\left(\ell_{k, p}+\frac{1}{2}\right)}
\end{aligned}
$$

where $\ell_{k, p}$ are defined analogously to eq. (4.43) and attached to each point of the $p$ space. $\sum_{S_{p}}$ is the sum over all representations at all points. In the continuum limit it becomes a fermionic path integral. $M_{R\left[\otimes S_{p}\right]}^{\left[\otimes S_{p}\right]}$ plays the role analogous to the corresponding factor in eq. (4.41) making the system highly non-trivial. It gives rise to the same selection rule (3.14) for allowed representations $R$ as in the one-dimensional case.

We hope to come back to this problem in the future and in this paper we propose eqs. (7.9), (7.10) as a definition of a $\mathrm{D}+1$-dimensional bosonic string theory directly in the continuous limit, as it was discussed in the introduction.

Another interesting question is the role of D-dimensional partition functions in higher representations. They might describe vortex-like excitations (i.e. monopole) which might 
be quite important for the physics of strings. It is not clear whether they are separated by a gap from the singlet vacuum.

\section{Discussion.}

The aim of this paper was to demonstrate the possibility to extract physical information about $1 D$ bosonic string by means of the analytical continuation of quantities known for the standard matrix oscillator. It was shown that not only the singlet (with respect to the $U(N)$ symmetry of the model) but also the adjoint partition functions can be obtained in the double scaling limit with the help of this procedure. It was shown that the adjoint partition function describes the one-vortex-anti-vortex-pair sector of the $X Y$-model coupled to gravity. It enabled us to calculate the critical temperature for the Berezinski-Kosterlitz-Thouless phase transition.

However, the method of analytical continuation obviously suffers from ambiguities which appear because the high symmetry of the matrix oscillator cannot be preserved in the upside-down case. Any cut-off imposed in order to stabilize the system decreases this symmetry. Therefore we had to guess a dependence on the cut-off using close analogy between the singlet and adjoint partition functions. However, in the case of higher representations, one apparently needs some microscopic information in order to make the continuation unambiguous. To begin with, one needs e.g. the decomposition of the sum over all representations with respect to the contributions of particular vortex-anti-vortex combinations. It may be done matching the partition functions with propagators of nonsinglet states as it was done in this paper for the adjoint representation. Another useful trick would be to introduce a regularization similar to one proposed in ref. [11] in the singlet case.

It is tempting to interpret the non-local matrix variable

$$
\Omega(0, \beta)=\widehat{T} \exp i \int_{0}^{\beta} d t A(t)
$$

as an operator creating a vortex of the unit charge. It would be interesting to include the angular degrees of freedom in the collective field representation [22] as well as eigenvalues.

Although, knowing the partition function for one vortex-anti-vortex pair, one can correctly estimate the position of the Berezinski-Kosterlitz-Thouless phase transition, in order to get a solution at the critical point, one should sum over all or, maybe, over the 
most important representations. This problem is far from being solved. However, it seems that properties of the $X Y$-model interacting with $2 \mathrm{D}$ quantum gravity are quite similar to those of the $X Y$-model on the plane: for $\beta>4 \pi$ one has the dilute-gas-of-dipoles phase, where a vortex and an anti-vortex are tied together forming a dipole; and for $\beta<4 \pi$ one has the plasma phase, where interactions between vortices are screened and an emerging finite correlation length leads to the change of the matter central charge from $c=1$ to $c=0$.

The model under investigation is the first example of a matrix model where the angular degrees of freedom (and not just the eigenvalues) of the matrix field play an important role. Now, it is clear that the angular degrees of freedom describe vortices and hence they are of a great concern in many physical applications. They become crucial when the central charge is bigger than 1. Apparently, one has to pay much more attention to them in order to understand the nature of this phase of the string theory from the matrix model point of view.

\section{Aknowledgements}

The authors are very indebted to E.Brezin and I.K.Kostov for many useful discussions and inspiring proposals on the early stage of this work. We also thank L.AlvarezGaume, J.-M.Daul, D.Gross, V.Fateev, A.Kirillov, I.Klebanov, A.Migdal, F.Smirnov, A.B.Zamolodchikov, Al.B.Zamolodchikov and especially M.Douglas for many valuable comments. 


\section{Appendix A.}

In this appendix we derive the selection rule (3.14) for representation contributing to the sum (3.15) and discuss the behavior of eigenfunctions of the Hamiltonian (3.16) with respect to permutations of the coordinates $z_{i}$. The Fourier decomposition of $\Psi$-functions of the original matrix Schroedinger equation gives rise to the expansion of the Green function (defined in the case of the harmonical oscillator by eqs. (4.14) and (4.15))

$$
G(X, Y)=\sum_{\{R\}} \sum_{a, b=1}^{d_{R}} g_{a b}^{R}(\lambda, \mu) D_{a b}^{R}(U)
$$

where $\lambda$ and $\mu$ are eigenvalues of $\mathrm{N} \times \mathrm{N}$ matrices $X$ and $Y\left(X=\Omega_{1} \lambda \Omega_{1}^{+}, Y=\Omega_{2} \mu \Omega_{2}^{+}\right)$ and $U=\Omega_{1}^{+} \Omega_{2} \in \mathrm{U}(\mathrm{N}) ; D_{a b}^{R}(U)$ is a matrix element in the space of a representation $R$. The symmetry

$$
G\left(X, \Omega Y \Omega^{+}\right)=G\left(\Omega^{+} X \Omega, Y\right)
$$

means that eq.(A1) is invariant under left and right shifts of $U$ by a diagonal unitary matrices: $U_{k l} \rightarrow U_{k l} e^{i\left(\theta_{k}-\varphi_{l}\right)}$. By definition,

$$
g_{a b}^{R}(\lambda, \mu)=\int(d U) G\left(\lambda, U \mu U^{+}\right) \bar{D}_{a b}^{R}(U)
$$

and, from the invariance of the Haar measure under this symmetry,

$$
\begin{aligned}
g_{a b}^{R}(\lambda, \mu) & =\int(d U) G\left(\lambda, U \mu U^{+}\right) \bar{D}_{a^{\prime} b^{\prime}}^{R}(U) \prod_{k}^{N} \frac{d \theta_{k}}{2 \pi} \bar{D}_{a a^{\prime}}\left(e^{i \theta}\right) \int_{0}^{2 \varphi} \prod_{k}^{N} \frac{d \varphi k}{2 \pi} \bar{D}_{b^{\prime} b}\left(e^{-i \varphi}\right) \\
& =P_{a a^{\prime}}^{R} g_{a^{\prime} b^{\prime}}^{R}(\lambda, \mu) P_{b^{\prime} b}^{R}
\end{aligned}
$$

where $P_{a b}^{R}=\int_{0}^{2 \pi} \prod_{k=1}^{N} \frac{d \theta_{k}}{2 \pi} \bar{D}_{a b}\left(e^{i \theta}\right)$ is a projector , since

$$
\begin{aligned}
\left(P^{R}\right)_{a b}^{2} & =\int_{0}^{\pi} \prod_{k} \frac{d \theta_{k}}{2 \pi} \int_{0}^{2 \pi} \prod_{k} \frac{d \varphi_{k}}{2 \pi} D_{a a^{\prime}}\left(e^{i \theta}\right) D_{a^{\prime} b}\left(e^{i \varphi}\right) \\
& =\int_{0}^{2 \pi} \prod_{k} \frac{d \theta_{k}}{2 \pi} \int_{0}^{2 \pi} \prod_{k} \frac{d \varphi_{k}}{2 \pi} D_{a b}\left(e^{i(\theta+\varphi)}\right)=P_{a b}^{R}
\end{aligned}
$$

The dimension of the subspace it projects on to is equal to

$$
d_{R}^{(0)}=\operatorname{tr}_{R} P^{R}=\int_{0}^{2 \pi} \prod_{k=1}^{N} \frac{d \theta_{k}}{2 \pi} \chi_{R}\left(e^{i \theta}\right)
$$


Under permutations of $\lambda\left(\lambda \rightarrow \rho \lambda \rho^{-1}\right)$ the Fourier coefficients behave as follows

$$
\begin{aligned}
g_{a b}^{R}\left(\rho \lambda \rho^{-1}, \mu\right) & =\int(d U) G\left(U^{+} \rho \lambda \rho^{-1} U, \mu\right) \bar{D}_{a b}^{R}(U) \\
& =\bar{D}_{a a^{\prime}}^{R}(\rho) g_{a^{\prime} b}^{R}(\lambda, \mu)
\end{aligned}
$$

and analogously for $\mu$

$$
\begin{aligned}
& g_{a b}^{R}\left(\lambda, \rho \mu \rho^{-1}\right)=\int(d U) G\left(\lambda, U \rho \mu \rho^{-1} U^{+}\right) \bar{D}_{a b}^{R}(U)= \\
& g_{a b^{\prime}}^{R}(\lambda, \mu) \bar{D}_{b^{\prime} b}^{R}\left(\rho^{-1}\right)=D_{b b^{\prime}}^{R}(\rho) g_{a b^{\prime}}^{R}(\lambda, \mu)
\end{aligned}
$$

It is clear from eqs. (A7) and (A8) that $g_{a b}^{R}(\lambda, \mu)$ is a tensor operator with respect to the permutations, i.e. the Weyl group. It means that properties of the Green function (or the $\Psi$-functions) with respect to the permutations are determined by the matrix elements of the Weyl group, which are non-trivial in general. For self-consistency the subspace defined by eqs.(A4) - (A6) has to be invariant under all permutations of $\lambda$ and $\mu$. It is not difficult to show that it is the subspace of all zero-weight vectors in the space of a representation which form an orbit with respect to the Weyl group.

All vectors in the space of a representation can be numbered by the Gel'fand-Zetlin patterns:

$$
\begin{aligned}
& \begin{array}{llll}
m_{1, N} m_{2, N} & \ldots & \ldots & m_{N-1, N} m_{N, N}
\end{array} \\
& m_{1, N-1} m_{2, N-1} \ldots \ldots m_{N-2, N-1} m_{N-1, N-1} \\
& \text {.. } \quad \ldots \\
& m_{13} m_{23} m_{33} \\
& m_{12} m_{22} \\
& m_{11}
\end{aligned}
$$

The rows in eq. (A9) are the highest weights in the following sequence of embeddings [19]

$$
\mathrm{U}(\mathrm{N}) \supset \mathrm{U}(\mathrm{N}-1) \supset \ldots \quad \ldots \supset \mathrm{U}(2) \supset \mathrm{U}(1)
$$

where $\mathrm{U}(k)$ is spanned by the first $k$ rows and columns of the $\mathrm{U}(\mathrm{N})$ matrix in the fundamental representation. The first row in eq. (A9) coincides with the $\mathrm{U}(\mathrm{N})$ highest weight and is fixed. Other numbers are restricted by the single condition : $m_{k, l} \geq m_{k, l-1} \geq m_{k+1, l}$. The character of $\mathrm{U}(1)$ is equal to $\exp \left(\mathrm{im}_{11} \varphi_{1}\right)$ and, hence, in order to get a non-zero answer in eq. (A6) one has to take $m_{11}=0$. From the identity $\mathrm{U}(k)=\mathrm{U}(1) \times \mathrm{SU}(k)$, it follows that 
$\chi_{u(k)}\left(e^{i \varphi}\right)=\chi_{s u(k)}\left(e^{i \varphi}\right) \exp i \sum_{j=1}^{k} m_{j, k}\left(\varphi_{1}+\varphi_{2}+\ldots+\varphi_{k}\right)$ and one has to take the trivial representation for all such $\mathrm{U}(1)$ subgroups:

$$
r_{k}=\sum_{j=1}^{k} m_{n, k}=0
$$

Eq (A11) has to be fulfilled for all $k \leq N$ and corresponding vectors will have the zero weight (by definition, the weight of a vector is equal to $\left[r_{1}, r_{2}-r_{1}, \ldots, r_{N}-r_{N-1}\right]$ ). When $k=N$, eq. (A11) gives the selection rule (3.14).

\section{Appendix B.}

Using the decomposition (3.1) and the eqs.(3.2), (3.3), we can represent (4.23) (in the functional integral formalism $(2.1),(2.2)$, but with free boundary conditions for $\varphi(0)$ and $\varphi(\beta))$ as

$$
K_{\alpha_{1} \alpha_{2}}(\beta)=\sum_{m, n=1}^{N}\left\langle e^{\alpha_{1} z_{m}(0)}\left|\left(\Omega(0) \Omega^{+}(\beta)\right)_{m n}\right|{ }^{2} e^{\alpha_{2} z_{n}(\beta)}\right\rangle
$$

Note that, for the free boundary conditions, the constraint (3.5) is absent due to the linear measure for the hermitian matrix field, $A(t)$, with the Gaussian weight (see eq.(3.2)). This functional integral is ultra-local in time, and one has only to take care of time ordering. The Vandermonde determinants of the z-variables cancel one another after the integration over $A(t)$ such that only two of them remain at the end points (see [1])

As the result we get

$$
\begin{aligned}
& K_{\alpha_{1} \alpha_{2}}(\beta)=\sum_{m, n=1}^{N} \int \prod_{k=1}^{N}\left[d z_{k}(t) e^{-\frac{1}{N} \operatorname{tr} \int_{-\infty}^{\infty} d t\left(\frac{1}{2} \dot{z}_{k}^{2}+N^{2} V\left(z_{k}\right)\right)}\right] \Delta(z(-\infty)) \Delta(z(\infty)) \\
& \times e^{\alpha_{1} z_{m}(0)+\alpha_{2} z_{n}(\beta)}\left(\operatorname{Texp}\left\{-\frac{1}{2 N} \int_{0}^{\beta} d t \widehat{Q}(z)\right\}\right)_{m n}^{m n}
\end{aligned}
$$

where the matrix $\widehat{Q}(z)$ is of the form

$$
\widehat{Q}(z)_{i j}^{m n}=\delta^{m n} \delta_{i j}\left(\sum_{k \neq i} \frac{1}{\left(z_{i}-z_{k}\right)^{2}}+\sum_{k \neq n} \frac{1}{\left(z_{k}-z_{n}\right)^{2}}\right)-2 \frac{\delta_{i}^{m} \delta_{j}^{n}}{\left(z_{i}-z_{j}\right)^{2}}
$$

where the double indices $(i, m)$ and $(j, n)$ represent ones in the space of the adjoint representations. The unitarity condition, $\Omega \Omega^{+}=I$, leads to the equations

$$
\sum_{i=1}^{N} \widehat{Q}_{i j}^{i n}=\sum_{j=1}^{N} \widehat{Q}_{i j}^{m j}=0
$$


hence the number of independent indices is equal to the dimension of the adjoint, $d_{\text {adj }}=$ $N^{2}-1$.

In the Hamiltonian language eqs. (B2), (B3) are equivalent to eqs. (4.26), (4.27) (where it was already taken $V(z)=-\frac{z^{2}}{2}$ ).

\section{Appendix C.}

One has to calculate the sum over ordered integer numbers of the type

$$
\Xi_{R}(q)=\sum_{n_{i} \geq n_{i+1}} M_{R \otimes S}^{S} \quad q^{\sum_{i=1}^{N} n_{i}}
$$

where $n_{i}$ are the components of the highest weight of a representation S. The coefficients $M_{R \otimes S}^{S}$ can be found by the following graphical rules [21]. Let us draw the vertical line and place the Young tableau of a representation $R$ in such a way that the number of boxes to the right of the line equals to the empty space to the left. After that one has to add all boxes of the tableau of a representation $S$ to the first tableau $(R)$ so that to reproduce the second one $(S)$ to the right of the line according to the following algorithm. One has to place all boxes from the first row of the second tableau in different columns of the first one so that they have formed a possible Young tableau. Then one has to proceed with boxes from the second row but with the restriction that above and to the right of each box in an obtained tableau the number of them must not exceed the number of boxes from the first row. Then one has to repeat this procedure for all rows. It is convenient to attach labels to all boxes of the second tableau, boxes from the same row having the same labels. Then $M_{R \otimes S}^{S}$ will be equal to the number of all possible combinations of labels. The structure of labeling is not fixed only in columns in which there are boxes of the first Young tableau. Therefore, one can consider every labeling in these columns as a separate sum over $n_{i}$-s contributing to eq.(C1) which takes in this case the form

$$
\Xi_{R}(q)=\sum_{\{L\}} \sum_{\left\{n_{i}\right\}} q^{\sum_{i=1}^{N} n_{i}}
$$

where $\sum_{\{L\}}$ is the sum over all possible labelings; $\sum_{\left\{n_{i}\right\}}$ is the sum over N integer numbers corresponding to all possible tableaux compatible with a particular labeling from the first sum. All numbers run to the infinity but from below the sum is restricted by a condition more complicated than the simple ordering. Nevertheless this sum gives just

$$
q^{a(L)} \Xi_{\text {sing }}(q)
$$


with an integer $a(L)$, hence eq.(4.50) is true and

$$
\begin{gathered}
P_{R}(q)=\sum_{\{L\}} q^{a(L)} \\
a(L)=\sum_{k=1}^{N} k \Delta_{k}
\end{gathered}
$$

where $\Delta_{k}=\min \left(n_{k}-n_{k+1}\right),\left(n_{N+1}=0\right)$ are minimal differences between the indices in the second sum in eq. $(\mathrm{C} 2)$.

If all labels less than $\mathrm{k}$ have been already placed in some way, one has a configuration which is equivalent to the case when the number of rows is equal to $N-k$ and placing k-labels one gets a number of configurations with the number of rows less by 1 . It gives rise to recurrence relations between contributions to $P_{R}(q)$ from different tables with the decreasing number of rows. For example, for the sequence of representations defined by eq. (4.52) there are only two ways to place $k$-labels:

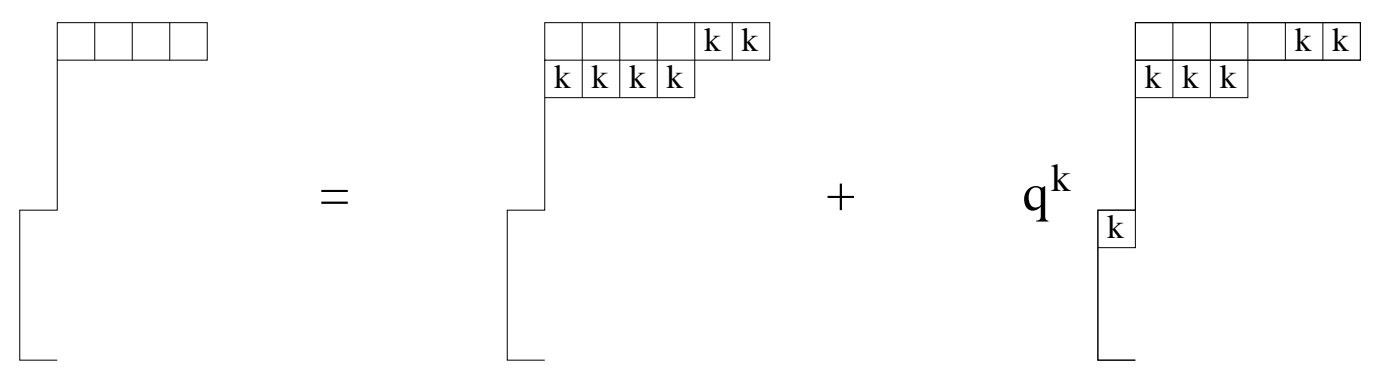

$$
a_{k}^{(n)}=a_{k+1}^{(n)}+q^{k} a_{k+1}^{(n-1)}
$$

and $P_{A_{n}}(q)=a_{1}^{(n)}$. Eq. (4.54) is a direct consequence of eq. (C6). For representations (4.53), the recurrence relation is a little bit more complicated: 


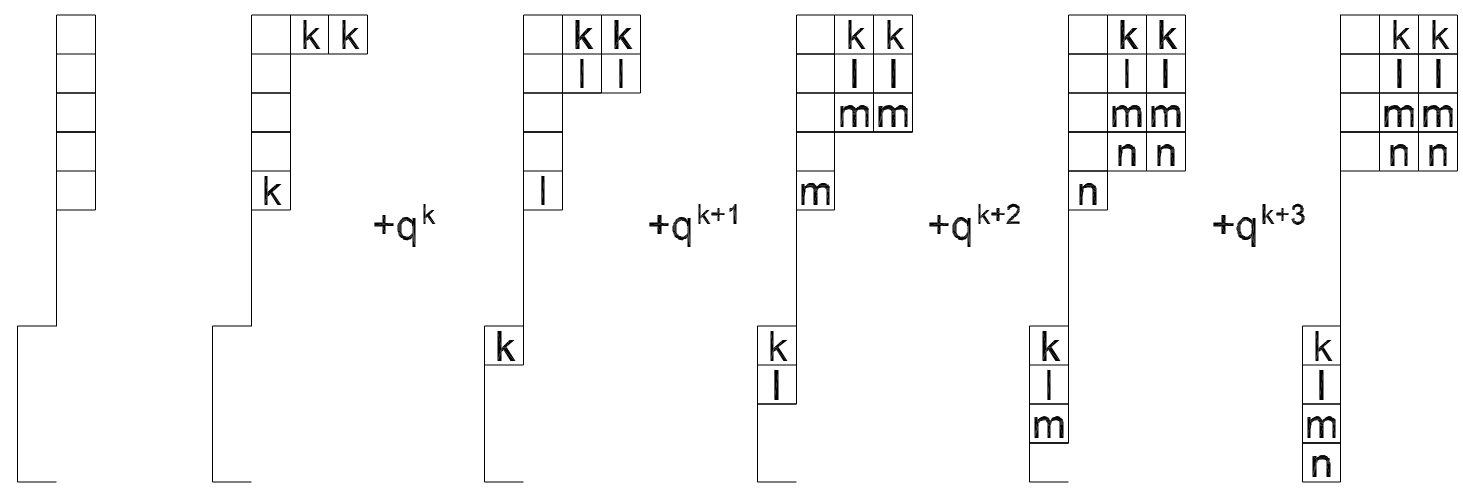

$$
b_{k}^{(n)}=\sum_{i=0}^{n} q^{i k} b_{k+1}^{(n-i)}
$$

and $P_{B_{n}}(q)=b_{1}^{(n)}(q)$. It is clear that eq. (C6) describes fermions (no more than one particle can fill the $k$ 'th level) and eq. (C7) describes bosons (the number of particles are not restricted). One can proceeds in the same way for other representations but the structure of answers will be the more complicated the more non-trivial Young tableaux are. Nevertheless, they can be always interpreted as statistical sums for $n$ particles in the equidistant level system, where $n$ is the number of positive (or negative) components of a highest weight. For example, for the sequence of representations

$$
C_{n}=(\underbrace{1, \ldots, 1}_{n}, 0, \ldots, 0, \underbrace{-1, \ldots,-1}_{n})
$$

one gets the recurrence relation: 


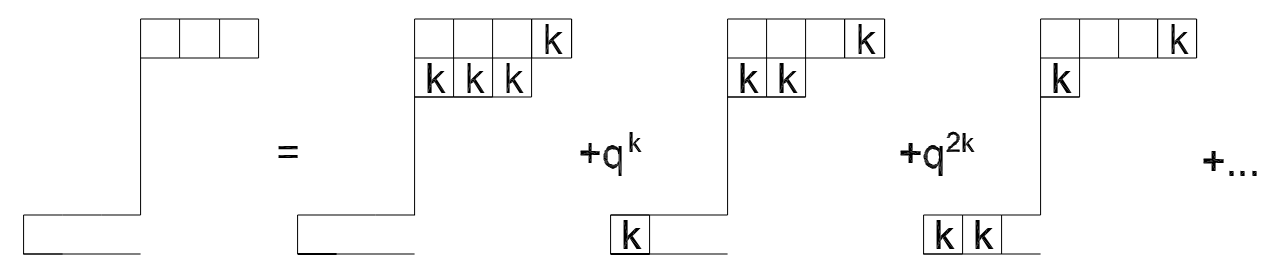

$$
c_{k}^{(n)}=c_{k+1}^{(n)}+\sum_{i=1}^{n} q^{k+i-1} c_{k+i+1}^{(n-i)}
$$

which describes fermions but with "wrong" weights: the weight of a fermion on the $k$-th level is equal to $q^{k-j}$ where $j=0$ if the $(k-1)$-th level is empty and $j=k-1$ otherwise. The form of the polynomials for several simplest representations are: i) for the adjoint

$$
P_{a d j}(q)=\frac{q-q^{N}}{1-q}
$$

ii) for 4 representation with $n=22$

$$
\begin{aligned}
& P_{A_{2}}=\frac{q^{3}\left(1-q^{N-2}\right)\left(1-q^{N-1}\right)}{(1-q)\left(1-q^{2}\right)} \\
& P_{B_{2}}=\frac{q^{2}\left(1-q^{N-1}\right)\left(1-q^{N}\right)}{(1-q)\left(1-q^{2}\right)} \\
& P_{C_{2}}=\frac{q^{2}\left(1-q^{N-3}\right)\left(1-q^{N}\right)}{(1-q)\left(1-q^{2}\right)}
\end{aligned}
$$

\section{References}

1. V.A.Kazakov, preprint LPTENS 90/30, December 1990;

2. D.Gross and I.Klebanov, Nucl.Phys. B344 (1990) 475;

3. D.Gross and I.Klebanov, Nucl.Phys. B354 (1990) 459;

\footnotetext{
2 the representation conjugate to $A_{2}$ gives the same answer.
} 
4. Marchesini and Onofri, J.Math.Phys. 21 (1980) 1103;

5. E.Brezin, C.Itzykson, G.Parisi and J.-B.Zuber, Comm.Math.Phys. 59 (1978) 35;

6. V.A.Kazakov and A.A.Migdal, Nucl.Phus. B311 (1988) 171;

7. E.Brezin, V.A.Kazakov and Al.B.Zamolodchikov, Nucl.Phys. B338 (1990) 673;

8. D.Gross and N.Miljkovic, Phys.Lett. B238 (1990) 217;

9. P.Ginsparg and J.Zinn-Justin, Phys.Lett. B240 (1990) 333;

10. J.Parisi, Phys.Lett. B238 (1990) 209, 213;

11. G.Moore, preprint RU-91-12 (1991), Nucl.Phys. B368 (1992) 557;

12. I.Kostov, Phys.Lett. B215 (1988) 499;

13. V.L.Berezinski, JETP 34 (1972) 610;

14. J.M.Kosterlitz and D.J.Thouless, J.Phys. C6 (1973) 1181;

15. J.Villain, J.Phys. C36 (1975) 581;

16. C.Itzikson and J.B.Zuber, J.Math.Phys. 21 (1980) 411;

17. D.P.Zelobenko, "Compact Lie Groups and Their Applications", Nauka 1970,Moscow (in Russian); Amer.Math.Soc.Translations, 40 (1973);

18. Y.Nambu, "Fermions living in a space of Lie group", Enrico Fermi Inst. preprint (1984);

19. A.N.Kirillov, J.Geom.and Phys. 5 (1988) 365;

20. A.O.Barut and R.Raczka, "Theory of Group Representations and Applications", World Scientific 1986,Singapore, Ch. 10, §1;

21. ibid, Ch. 8, §8;

22. S.Das and A.Jevicki, Mod.Phys.Lett. A5 (1990) 1639. 